\title{
Revisione degli Scotonomus della Sardegna e descrizione di dodici specie nuove (Coleoptera Staphylinidae)
}

\begin{abstract}
Riassunto - Sulla base di copioso materiale raccolto durante ricerche protrattesi per lunghi anni su tutto il territorio regionale e dello studio di tutti i tipi e degli esemplari reperibili nei principali musei italiani ed europei, è stata effettuata la revisione delle specie del genere $S c o t o-$ nomus Fauvel, 1873 della Sardegna. Vengono descritte 12 specie nuove (S. terteniensis sp. n., S. bifidus sp. n., S. kraussei sp. n., S. barbaricinus sp. n., S. mirificus sp. n., S. baroniae sp. n., S. caneparii sp. n., S. lulensis sp. n., S. dorgaliensis sp. n., S. confusus sp. n., S. montalbicus sp. n., S. fauveli sp. n.) per cui quelle attualmente note assommano a 21; viene proposta la seguente sinonimia: Scotonomus doderoi Binaghi, 1970 = Scotonomus troglophilus Coiffait, 1982, syn. n.; è designato il lectotypus di Scotonomus raymondi Fauvel; viene smentita la sinonimia tra $S$. raymondi e $S$. doderoi, qui ritenute specie distinte; vengono individuate ben 5 specie differenti, alcune nuove per la Scienza, nella serie tipica di S. doderoi; gli Scotonomus sardi vengono divisi in tre gruppi di specie, è proposta una chiave dicotomica per l'identificazione dei taxa e di tutte le specie vengono forniti i disegni originali dell'edeago e le carte di distribuzione.
\end{abstract}

\begin{abstract}
Revision of the Scotonomus from Sardinia with description of twelve new species. On the basis of aboundant material collected during researchs that lasted for many years throughout the region and the study of all types and specimens preserved in the main Italian and European museums, it is proposed the revision of the species of Scotonomus Fauvel of Sardinia. Twelve new species are described (S. terteniensis sp. n., S. bifidus sp. n., S. kraussei sp. n., S. barbaricinus sp. n., S. mirificus sp. n., S. baroniae sp. n., S. caneparii sp. n., S. lulensis sp. n., S. dorgaliensis sp. n., S. confusus sp. n., S. montalbicus sp. n., S. fauveli sp. n.); currently 21 species of Scotonomus are known of Sardinia; the following synonymy is established: Scotonomus doderoi Binaghi, $1970=$ Scotonomus troglophilus Coiffait, 1982, syn. n.; the lectotype of $S$. raymondi Fauvel is designated; synonymy of $S$. raymondi and $S$. doderoi Binaghi, that are distinct species, is rejected; five different species, some new to science, are recognized in the typical series of $S$. doderoi; species of Sardinia are divided into three groups and key to their identification is proposed; all species are provided of original figures of the aedeagus and distributional maps.
\end{abstract}

Key words: Staphylinidae, Paederinae, Scotonomus, Sardinia, revision, new species, synonymy, lectotype.

\section{INTRODUZIONE}

Il genere Scotonomus Fauvel, 1873, endemico dell'Italia centro-meridionale e della Sardegna, è stato oggetto di studio da parte di un ristretto numero di autori (Binaghi, 1970; Coiffait, 1971, 1982; Pace, 1977; Zanetti, 1977; Bordoni, 1975, 2006, 2010; Bordoni \& Magrini, 2003).

Si tratta di Stafilinidi che vivono in ambiente ipogeo, rinvenibili soprattutto sotto pietre profondamente infossate e talora anche nella parte superficiale del suolo in zone forestali. Essi hanno caratteri esoscheletrici molto omogenei, per cui le specie sono assai simili tra loro, depigmentate, attere, anoftalme (quasi sempre è presente un solo ommatidio appena visibile a forte ingrandimento). I maschi presentano una vistosa e stretta incisione al lato ventrale del sesto sternite visibile mentre le femmine hanno il medesimo sternite con margine posteriore vistosamente arrotondato.

A causa della notevole somiglianza esteriore, è praticamente quasi inutile una descrizione particolareggiata della morfologia esterna (i caratteri forniti nelle descrizioni sono quindi da intendersi indicativi) mentre è essenziale lo studio dell'edeago; questo è composto da un bulbo basale dal quale si dipartono $i$ parameri che recano sulla metà distale un certo numero di setole (tre apicali e altre subapicali, dirette verso l'interno nelle specie dell'Italia centrale e verso l'esterno in quelle dell'Italia meridionale e Sardegna). Dal bulbo basale dipartono 3 lamelle copulatrici (preferiamo adottare questa semplice terminologia) nelle specie continentali o 4 nelle specie della Sardegna, di forma e disposizione differente nelle varie specie e quindi di fondamentale importanza per lo studio dei singoli taxa.

Come già evidenziato (Bordoni \& Magrini, 2003), occorre che la lettura di tali lamelle sia facilitata da disegni accurati e di adeguata grandezza (quelli proposti, ad esempio, da Binaghi nel 1970 sono scarsamente efficaci), poiché esse si presentano molto vicine tra loro e normalmente sovrapposte. E' quindi necessario far bollire l'edeago (per pochi istanti, onde evitare che le lamelle si discostino eccessivamente) in una soluzione di $\mathrm{KOH}$ al $10 \%$ e poi in acqua distillata, prima di passare l'organo in alcool a $70^{\circ}$, affinché le lamelle si puliscano e si distacchino 
almeno un poco tra loro. Così facendo è possibile l'esame dettagliato dell'organo, ruotato a piacimento in euparal e quindi disposto su un supporto trasparente, assieme all'esemplare. Questa tecnica consente di leggere con accuratezza la forma delle lamelle e di fornire una loro adeguata raffigurazione.

La distribuzione del genere è di tipo chiaramente paleotirrenico. Binaghi (1970) sosteneva che la Sardegna centrale, dove sono localizzate un gran numero di specie, costituisse il centro di diffusione del genere e che $S$. sardus, localizzato nella zona del Gennargentu, fosse da ritenersi la specie più "antica". Sempre secondo questo autore il genere sarebbe approdato sull'Italia tirrenica attraverso il ponte sardopontino, come già suggerito da Jeannel (1937) per il genere Rhegmatobius Jeannel, 1937 (Carabidae Trechinae). In questa sede ci limitiamo ad osservare che l'ipotesi di un "ponte sardo-pontino" non è contemplata nelle più recenti teorie zoogeografiche; a nostro parere l'attuale distribuzione del genere è spiegabile, come in numerosi altri casi, con le crisi di salinità del Messiniano. Anche l'elevato numero di taxa presenti in Sardegna non implica necessariamente che l'isola sia il centro di diffusione del genere: l'alta speciazione degli Scotonomus in Sardegna può essere semplicemente spiegata con la complessa orografia del territorio e le sue vicende paleogeografiche.

In seguito allo studio di un gran numero di esemplari, frutto di accurate ricerche protrattesi per lunghi anni su tutto il territorio regionale, siamo in grado con questo contributo di fornire un quadro molto più approfondito e ricco del popolamento della Sardegna, tramite anche l'esame e la ridescrizione dei tipi delle specie precedentemente descritte.

La specie tipo del genere è $S$. raymondi Fauvel, 1873 (da considerarsi specie tipo per monotipia), descritta su materiale di "Toscane (Baudi), Sardaigne, Sassari (Raymond)". In seguito Saulcy (1878) separa le popolazioni di Toscana descrivendole come S. etruscus. Binaghi (1970), conserva come loc. typ. di $S$. raymondi Fauvel la località di Sassari (Raymond), attribuendo però alla specie esemplari di varie altre località della Sardegna centro-settentrionale che, con il progredire degli studi (Coiffait, 1971 e hoc opus), sono risultati attribuibili ad altri taxa.

Nella sua revisione degli Scotonomus, Binaghi (1970) citava per la Sardegna le seguenti specie: S. sardus Fagel, 1969, S. simpatricus Binaghi, 1970, S. doderoi Binaghi, 1970 e S. raymondi Fauvel,
1873. Attualmente, considerando le specie successivamente descritte da altri autori (Coiffait, 1971; Bordoni, 2006; Bordoni \& Magrini, 2003), quelle descritte nel presente contributo e le nuove sinonimie, le specie note per la Sardegna ammontano a 21.

\section{ACRONIMI}

cB- coll. Bordoni, Firenze; cC- coll. Canepari, San Donato Milanese; cM- coll. Magrini, Firenze; cP- coll. Paladini, Vicchio; cS- coll. Schülke, Berlin; cT-coll. Tronquet, Moligt-les-Bains; DEI- Deutsches Entomologisches Institut, Müncheberg; IRSNB- Institut royal d'Histoire naturelle, Bruxelles; MCZMuseo civico di Zoologia, Roma; MHNG- Muséum d'Histoire naturelle, Genève; MNHNP- Museum national d'Histoire naturelle, Paris; MNHU- Museum für Naturkunde der Humboldt-Universität, Berlin; MSNG- Museo civico di Storia naturale "G. Doria", Genova; MSNM- Museo civico di Storia naturale, Milano; MZF- Museo Zoologico "La Specola", Firenze; MZUR- Museo di Zoologia dell’Università degli Studi "La Sapienza", Roma; NMW- Naturhistorisches Museum, Wien.

\section{TRATTAZIONE DELLE SPECIE}

Scotonomus sardus Fagel, 1969

Scotonomus sardus Fagel, 1969: 103; Binaghi, 1970: 123; Coiffait, 1971: 730; Bordoni, 2006: 7.

DesCrizione. Corpo lungo 4,5-5,1 mm. E' la specie di Scotonomus più grande e robusta presente in Sardegna. Capo largo, a lati largamente arrotondati; pronoto massiccio, dilatato anteriormente.

Edeago (Fig. 1) lungo 1,1-1,2 mm, con lamella mediana di forma molto caratteristica, con dente molto pronunciato e lungo. La lamella destra, in visione ventrale, appare molto stretta, rivolta verso la lamella mediana e inserita sotto la stessa. La lamella sinistra, ancora in visione ventrale, è robusta e larga. Lamella posteriore piuttosto corta, come si osserva anche in visione dorsale (Fig. 2).

Materiale tipico. Il tipo ô è etichettato "Aritzo, Sard., A. Dodero VI.1910", "G. Fagel det., sardus n. sp.", "Type" (a stampa su cartellino arancione) (IRSNB); paratipi [tutti con il cartellino arancione "Paratype" (a stampa), "Aritzo, Sardinia, A. Dodero

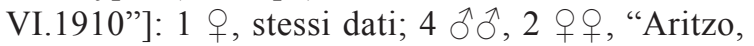
Sardinia", senza data (dei quali uno con il cartellino "Scotonomus raymondi var. major", uno con il car- 

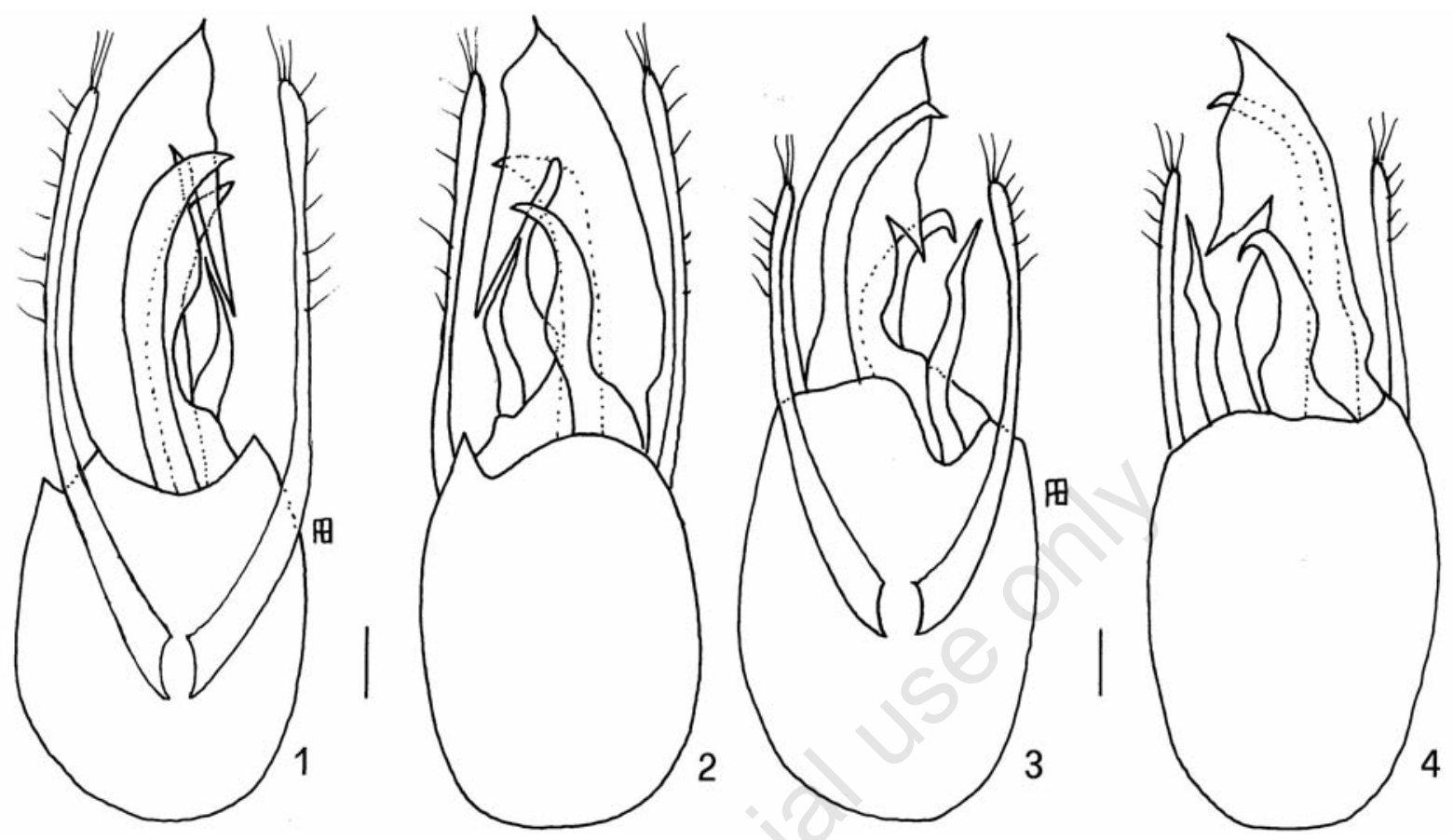

Figg. 1-4. Scotonomus sardus Fagel: 1- edeago in visione ventrale; 2 - in visione dorsale; Scotonomus terteniensis sp. n.: 3 edeago in visione ventrale; 4 - in visione dorsale. Scala: $0.1 \mathrm{~mm}$

tellino "Scotonomus etruscus raymondi", uno con il cartellino "etruscus") (IRSNB).

Materiale esaminato. Aritzo (Nuoro), Dodero leg. IV-VI.1901-1912, 8 ふึิึ, 2 우 (più altri 37 exx.); Aritzo, A. Dodero VI.1910, 6 exx. (NMW), 1 ex. (cB); Aritzo, A. Dodero, 5 exx. (NMW); Aritzo, 1 ex. (NMW); Aritzo, 1600 m, G. Krüger leg. V.1912, 10 exx. (DEI), 3 exx. (cB); Aritzo, Mt Gennargentu, G. Krüger leg., 3 exx. (DEI), 1 ex. (cB); Aritzo, Dodero leg., 1 ex. (DEI); Aritzo, 1 क; Aritzo, Dodero leg., 1 స̂ (MNHU), 3 exx. (MCZ), 1 ex. (cB); Aritzo, F. Solari leg. 10.VI.1910, 1 ô; Aritzo, F. Solari leg. 110.VI.1910, 1 ex. (MZUR), 1 ex. (MHNG); Gennargentu, (NU), Mt d'Iscudu, 1200 m, I. Bucciarelli 31.V.1972, 1 \& (MSNM); Gennargentu (Nuoro),

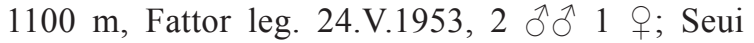
(Nuoro), Dodero leg. IV.1902 e V.1904, 2 춧 9 우우 (MSNG); stessi dati, A. Dodero leg. 2.V.1902, 2 우우 (DEI); Seui, A. Dodero, 1 ex. (NMW); Seui (Nuoro), M. Curti leg. III.1977, 1 ex.; Seui, Mt Arqueri, M. Curti leg. III.1979, 3 exx. (MHNG); Seulo, Seui (Nuoro), 400 m, M. Curti leg. 6.III.1979, 2 exx.
(MHNG), 1 ex. (cS); Mt Gresia, Aritzo (Nuoro), Sbordoni leg. 10.V.1967, 3 ठิ 1 ๆ (cB); Mt Chiesa (= Mt Cresia ovvero Mt Gresia), 1 o (cB), 1 우 (NMW); Ulassai (Ogliastra), Fancello leg. 3.I.2003, 1 ô; Sardinia, 1 ex. (cB).

DisTRIBUZIONE. Sardegna centrale: massiccio del Gennargentu meridionale e dintorni, tra 400 e $1100 \mathrm{~m}$.

\section{Scotonomus terteniensis sp. $\mathrm{n}$.}

Materiale esaminato. Holotypus $\widehat{\partial}$ : Sardegna, Tertenia (Ogliastra), loc. Tacchixeddu, $500 \mathrm{~m}$, Fancello \& Leo leg. 15.III.1990 (cB); paratypus: stessi dati, 1 ㅇ (cB); stessi dati, Fancello leg. 24.XII.1992, 1 우 (cC).

Descrizione. Corpo lungo 4,2 $\mathrm{mm}$. Molto più piccolo della media degli esemplari esaminati di $S$. sar$d u s$, con capo meno dilatato, più stretto anteriormente e a lati meno largamente arrotondati; pronoto meno dilatato in avanti, meno massiccio; punteggiatura di capo e pronoto un poco più fitta; elitre con microreticolazione poligonale più netta.

Edeago (Fig. 3) lungo 1,1 mm, simile a quello di $S$. sardus, con parameri proporzionalmente più 
corti che in $S$. sardus, con lamella mediana provvista di un dente molto più tozzo, con lamella destra, in visione ventrale, rivolta verso l'esterno, con lamella sinistra, ancora in visione ventrale, assai meno robusta e larga e con lamella posteriore più corta e meno robusta, come si osserva anche in visione dorsale (Fig. 4).

DistRIBUZIONE. Nota della sola località tipica, nel sud dell'Ogliastra, a sud- est del Gennargentu.

DeRIVATIO NOMinis. Il nome della specie fa riferimento alla località tipica.

Note. Si poteva ipotizzare che gli esemplari suindicati fossero riferibili ad una possibile variabilità della specie precedente ma si è ritenuto di considerarli appartenenti a specie distinta in base alle seguenti considerazioni: tutti gli esemplari maschi di S. sardus esaminati (oltre 40) hanno lamella mediana provvista di dente interno ugualmente strutturato, molto lungo e ad apice lungamente appuntito; il dente suddetto in S. terteniensis sp. n. è molto più corto, tozzo, largo e robusto; le altre lamelle, e quella sinistra in particolare, hanno forma differente; le dimensioni di S. terteniensis sono nettamente inferiori a quelle di $S$. sardus, tenendo conto della esigua lunghezza degli Scotonomus; tutti gli esemplari di S. sardus esaminati sono stati raccolti solo sul massiccio del Gennargentu; la zona di raccolta di $S$. terteniensis non fa più parte del Gennargentu ma si trova a bassa quota nell'Ogliastra, presso il mare.

Scotonomus bifidus sp. $\mathrm{n}$.

Materiale esaminato. Holotypus ${ }^{\top}$ : Sardegna, S. Niccolò Gerrei (Cagliari), loc. Pranu Sanguni, 550 m, Fancello \& Leo leg. 11.III.1995 (cB); paratypi: stessi dati, Fancello leg. 10.IV.1996, 1 † (cC); Perdasdefogu (Ogliastra), Fancello \& Leo leg.

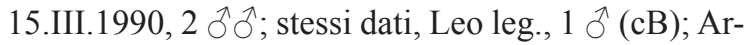
mungia (CA), dintorni Grotta Gospunu, 100 m, A. Paladini 6.III.2011, 1 ठ (cP).

DesCrizione. Corpo lungo 4,2 $\mathrm{mm}$. Molto più piccolo della media degli esemplari esaminati di $S$. sar$d u s$, con capo simile a quello della specie precedente; pronoto subrettangolare, non dilatato anteriormente, a lati subrettilinei e subparalleli; elitre un poco più corte che in $S$. sardus; punteggiatura simile a quella di $S$. sardus.

Edeago (Fig. 5) lungo $1 \mathrm{~mm}$, simile a quello di $S$. sardus, con parameri proporzionalmente più corti, con lamella mediana ad apice più prominente e dente biforcuto; lamella destra, in visione ventrale, assai più robusta e larga; lamella sinistra, ancora in visione ventrale, larga nella porzione mediana e molto lunga e stretta in quella apicale; lamella posteriore molto lunga e stretta, come si osserva anche in visione dorsale (Fig. 6).

Distribuzione. Sardegna sud-orientale (Gerrei e Ogliastra meridionale).

Derivatio Nominis. Prende nome dalla forma della lamella mediana dell'edeago, provvista di due denticolazioni.

Scotonomus kraussei sp. n.

Materiale esaminato. Holotypus ${ }^{\lambda}$ : Sardegna, Asuni (Oristano), Fancello \& Leo leg. 27.XII.1989 $(\mathrm{cB})$; paratypi: stessi dati, 1 ๆ $(\mathrm{cB})$; stessa località, Krausse leg., 1 ㅇ (MSNG).

DesCrizione. Corpo lungo 4,4 mm. Più piccolo della media degli esemplari esaminati di $S$. sardus, ma un poco più lungo delle due specie precedenti. Capo allungato, appena ristretto in avanti, a lati assai poco arrotondati; pronoto meno massiccio che in $S$. sar$d u s$, meno dilatato anteriormente; elitre più larghe; punteggiatura di capo e pronoto più fitta.

Edeago (Fig. 7) lungo $1,1 \mathrm{~mm}$, con lamella mediana simile a quella di $S$. sardus ma con apice di forma molto caratteristica e biforcuto come in $\mathrm{S}$. bifidus; lamella destra, in visione ventrale, a forma di amo, stretta e lunga; lamella sinistra, ancora in visione ventrale, molto grande, dilatata e larga, ad apice acuto; lamella posteriore lunga e stretta, ad apice uncinato, come si osserva anche in visione dorsale (Fig. 8).

Distribuzione. E' nota della sola località tipica nella Sardegna centro-occidentale (Oristanese).

Derivatio nominis. Specie dedicata a Anton Hermann Krausse (1878-1929) che per primo la raccolse. Krausse soggiornò in Sardegna dal 1906 al 1914, apportando notevole contributo alle conoscenze naturalistiche dell'isola (Cassola, 1983).

Note. L'olotipo, raccolto allo stato di resto, è mancante di pronoto ed elitre e l'edeago manca dei parameri, per cui la descrizione dei caratteri esterni si basa prevalentemente sulla femmina.

Scotonomus barbaricinus sp. n.

Materiale esaminato. Holotypus ỡ: Sardegna, De- 


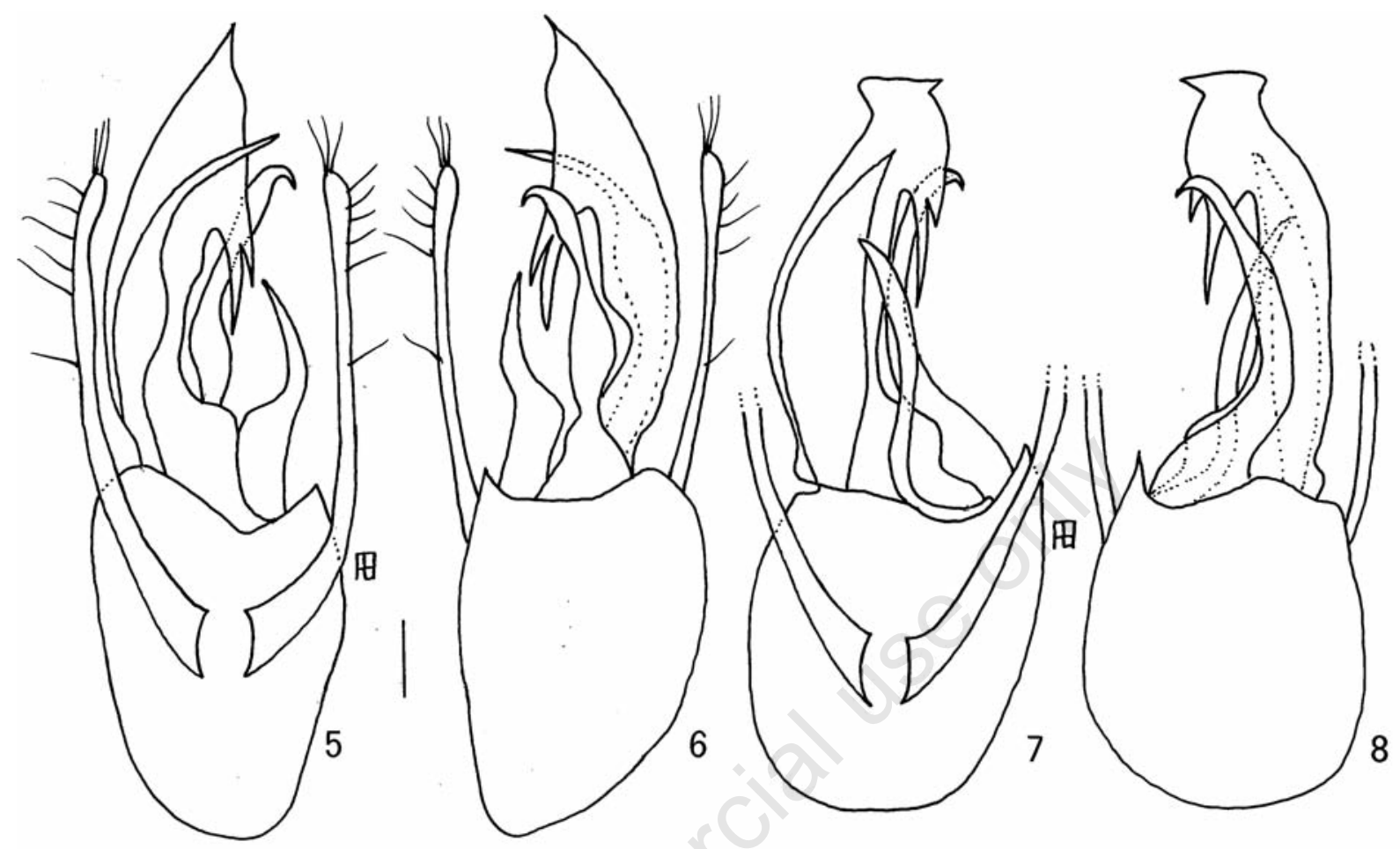

Figg. 5-8. Scotonomus bifidus sp. n.: 5 - edeago in visione ventrale; 6 - in visione dorsale; Scotonomus kraussei sp. n.: 7 - edeago in visione ventrale; 8 - in visione dorsale. Scala: $0.1 \mathrm{~mm}$

sulo (Nuoro), dint. di S'Arcu de Tascussi, $1200 \mathrm{~m}$, Leo leg. 24.V.1986 (cB); paratypus: stessi dati, 1 $(\mathrm{cB})$.

DESCRIZIONE. Corpo lungo $3,9 \mathrm{~mm}$. Molto più piccolo della media degli esemplari esaminati di S. sardus, con capo più lungo e meno dilatato, con lati meno arrotondati; pronoto più dilatato anteriormente e più stretto posteriormente che in S. sardus, con lati assai meno arrotondati; elitre con superficie più rugosa.

Edeago (Fig. 9) lungo 0,9 mm, simile a quello della specie precedente, ma con porzione apicale della lamella mediana diversamente conformata, non biforcuta, ma simile a $S$. sardus; lamella destra molto corta, non visibile in visione ventrale, in quanto posizionata posteriormente, come si osserva in visione dorsale (Fig. 10); lamella sinistra, ancora in visione ventrale, lunghissima e molto stretta; lamella posteriore stretta e lunga, ad apice uncinato.

DistribuZione. Nota solo della località tipica nel Gennargentu.

Derivatio NOMINIS. Il nome deriva dagli abitanti della Barbagia, regione storico-geografica della località tipica.

Scotonomus simpatricus Binaghi, 1970

Scotonomus simpatricus Binaghi, 1970: 124; Coiffait, 1982: 162.

Materiale tiPICo. Il tipo ồ è etichettato "Dorgali, Sardegna, A. Dodero VI.1910", "n. sp.", "Binaghi 1970, Holotypus" (in rosso su cartellino bianco di mano di Binaghi), "Holotypus (a stampa), Scotonomus simpatricus Binaghi 1970 (di mano di Binaghi)" su cartellino arancione (MSNG); paratipi [tutti con cartellino arancione "Paratypus (a stampa), Scotonomus simpatricus Binaghi 1970 (di mano di Bina-

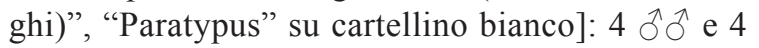
$\uparrow$, , stessi dati (MSNG); 1 ก e 1 ค , stessi dati, V.1910 (MZF).

Materiale esaminato. Sardinia, Dorgali, $1 \hat{\jmath}(\mathrm{cB})$. DesCrizione. Corpo lungo 4-4,2 mm; simile nell'aspetto generale a $S$. sardus ma sensibilmente più 
piccolo, con punteggiatura più rada e minuta, pronoto più stretto, elitre con superficie più lucida.

Edeago (Fig. 11) lungo 0,9 mm, con lamella mediana di forma caratteristica, con lamella destra, in visione ventrale, molto stretta, ad apice arrotondato, rivolta verso l'esterno; lamella sinistra, ancora in visione ventrale, stretta e ad apice acuto; lamella posteriore molto larga alla base, stretta nella porzione distale e con apice arrotondato, come si osserva anche in visione dorsale (Fig. 12).

DisTRIBUZIONE. E' specie nota solo della zona di Dorgali nel Nuorese orientale, a circa $400 \mathrm{~m}$ di quota, ove convive con $S$. doderoi.

\section{Scotonomus mirificus $\mathrm{sp}$. $\mathrm{n}$.}

Materiale esaminato. Holotypus $\delta^{\lambda}$ : Sardegna, Torpé (Nuoro), Brunella, 200 m, Fancello leg. 22.IV.2010 (cB).

Descrizione. Corpo lungo 4,2 mm. Capo dilatato posteriormente, con lati ben arrotondati; pronoto massiccio, anteriormente dilatato, con angoli anteriori largamente arrotondati e lati poco arrotondati; elitre molto corte; punteggiatura rada.

Edeago (Fig. 13) lungo $1 \mathrm{~mm}$, con lamella mediana di forma caratteristica, ad apice largo e fortemente arrotondato e a sinistra uncinato; lamella destra, in visione ventrale, spostata a contatto con la lamella mediana, molto lunga e molto stretta, ad apice acuto; lamella sinistra sinuosa, di larghezza variabile, ad apice acuto; lamella posteriore spostata verso l'esterno, un poco sinuosa e ad apice uncinato, come si osserva anche in visione dorsale (Fig. 14).

DistribuZione. Nota solo della località tipica, nel Nuorese nord-orientale, a nord del massiccio del Monte Albo, a poca distanza dalla costa.

Derivatio nominis. Dal latino mirificus (straordinario), in relazione alla forma del tutto particolare della lamella mediana dell'edeago.

\section{Scotonomus baroniae sp. $\mathrm{n}$.}

Materiale esaminato. Holotypus $\delta^{\lambda}$ : Sardegna, Lodé (Nuoro), Monte Tundu, 600 m, Fancello leg. 27.XI.2001 (cB).

DesCRIZIONE. Corpo lungo 4,4 mm; capo posteriormente molto dilatato, a lati molto arrotondati; pronoto massiccio, anteriormente dilatato e largo; elitre corte, con lati molto arrotondati; punteggiatura del pronoto piuttosto regolare e fitta.
Edeago (Fig. 15) lungo 0,9 mm, con lamella mediana molto larga alla base, ristretta verso l'apice che è uncinato; lamella destra accostata alla precedente, simile a quella ma più stretta e corta; lamella sinistra, ancora in visione ventrale, stretta e lunga, sinuosa, ad apice acuto; lamella posteriore spostata verso l'esterno, stretta, un poco dilatata nella porzione mediana, ad apice uncinato, come si osserva anche in visione dorsale (Fig. 16).

Distribuzione. Nota solo della località tipica, nel Nuorese nord-orientale, a nord-ovest del Monte Albo.

DERIVATIO NOMINIS. Dalla regione storico-geografica (Baronia) della località tipica.

\section{Scotonomus caneparii sp. n.}

Materiale esaminato. Holotypus $\delta^{\Uparrow}$ : Sardegna, Padru (Sassari), Sitagliacciu, 170 m, Fancello leg. 23.II.2000 (cB); paratypi: stessi dati, Fancello leg. III.2010, 1 (MSNG); stessi dati, Fancello leg. 10.IV.2010, 1 ô 1 of (cB); Orune (Nuoro), Nuraghe Nunnale, $500 \mathrm{~m}$, Fancello leg. 20.IV.2010, 1 ઈิ 1 q (cB).

Descrizione. Corpo lungo 4,2 mm; capo posteriormente dilatato, a lati arrotondati; pronoto massiccio, piuttosto ristretto posteriormente; elitre larghe con lati poco arrotondati; punteggiatura di capo e pronoto piuttosto fitta, rada sulle elitre.

Edeago (Fig. 17) un poco più lungo di 0,8 $\mathrm{mm}$, con lamella mediana robusta ma non particolarmente larga, prima della strozzatura subapicale, ad apice di forma particolare, con lamella destra, in visione ventrale, sovrapposta alla precedente, molto larga, stretta ed uncinata nella porzione apicale; lamella sinistra, ancora in visione ventrale, lunga e robusta, ad apice acuto e vistosamente rivolto all'esterno; lamella posteriore larga alla base, quindi stretta, un poco sinuosa, ad apice uncinato, come si osserva anche in visione dorsale (Fig. 18).

DistribuZione. Sardegna nord-orientale, tra Nuoro ed Olbia.

Derivatio Nominis. La specie è dedicata a Claudio Canepari, noto specialista di Coleoptera Coccinellidae, che ha fatto generosamente dono del tipo ad uno di noi.

Scotonomus lulensis sp. $\mathrm{n}$.

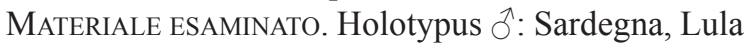
(Nuoro), Dodero VI.1911 (MSNG); paratypi: stessi 

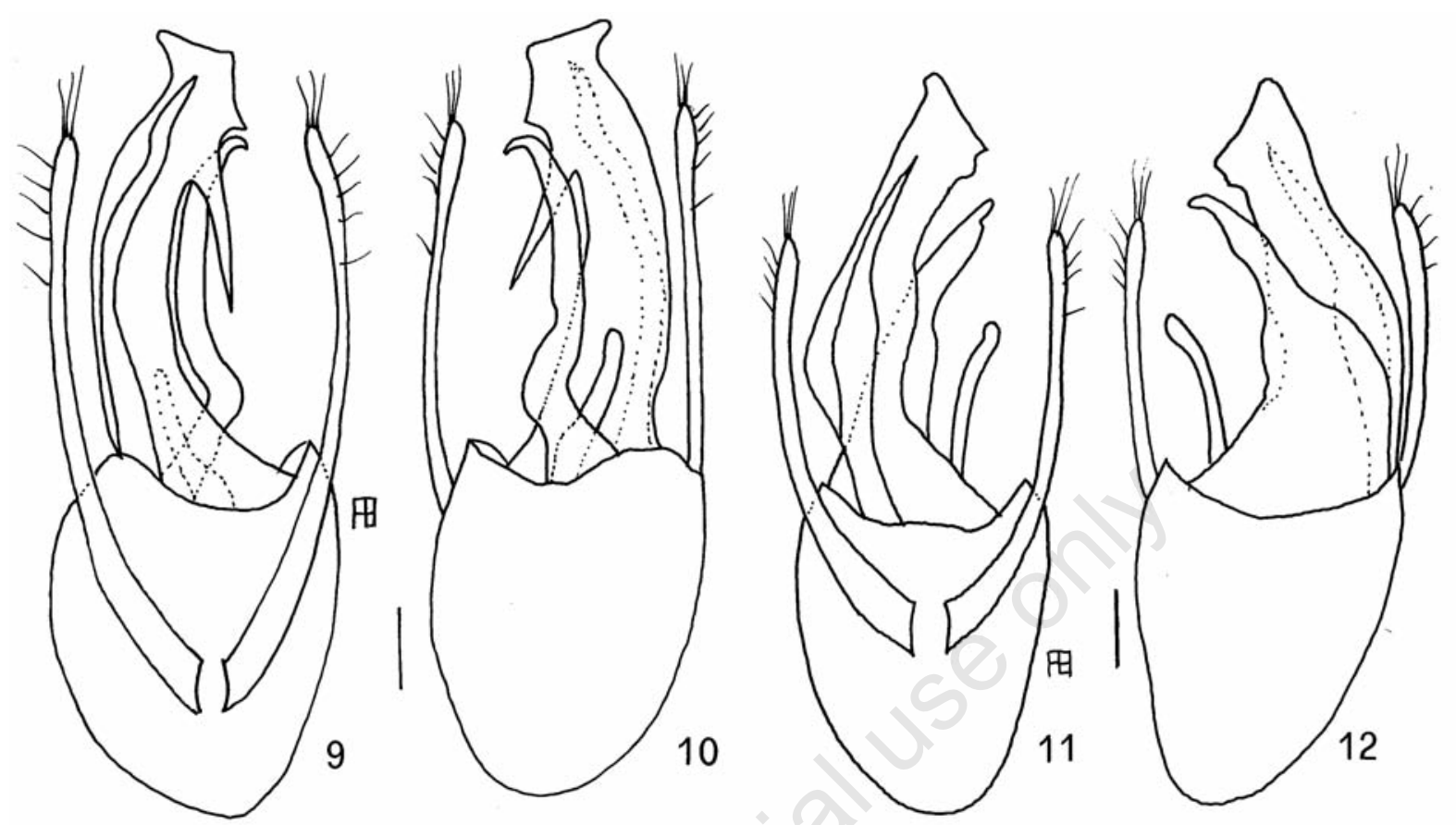

Figg. 9-12. Scotonomus barbaricinus sp. n.: 9 - edeago in visione ventrale; 10 - in visione dorsale; Scotonomus simpatricus $\mathrm{Bi}-$ naghi: 11 - edeago in visione ventrale; 12 - in visione dorsale. Scala: $0.1 \mathrm{~mm}$

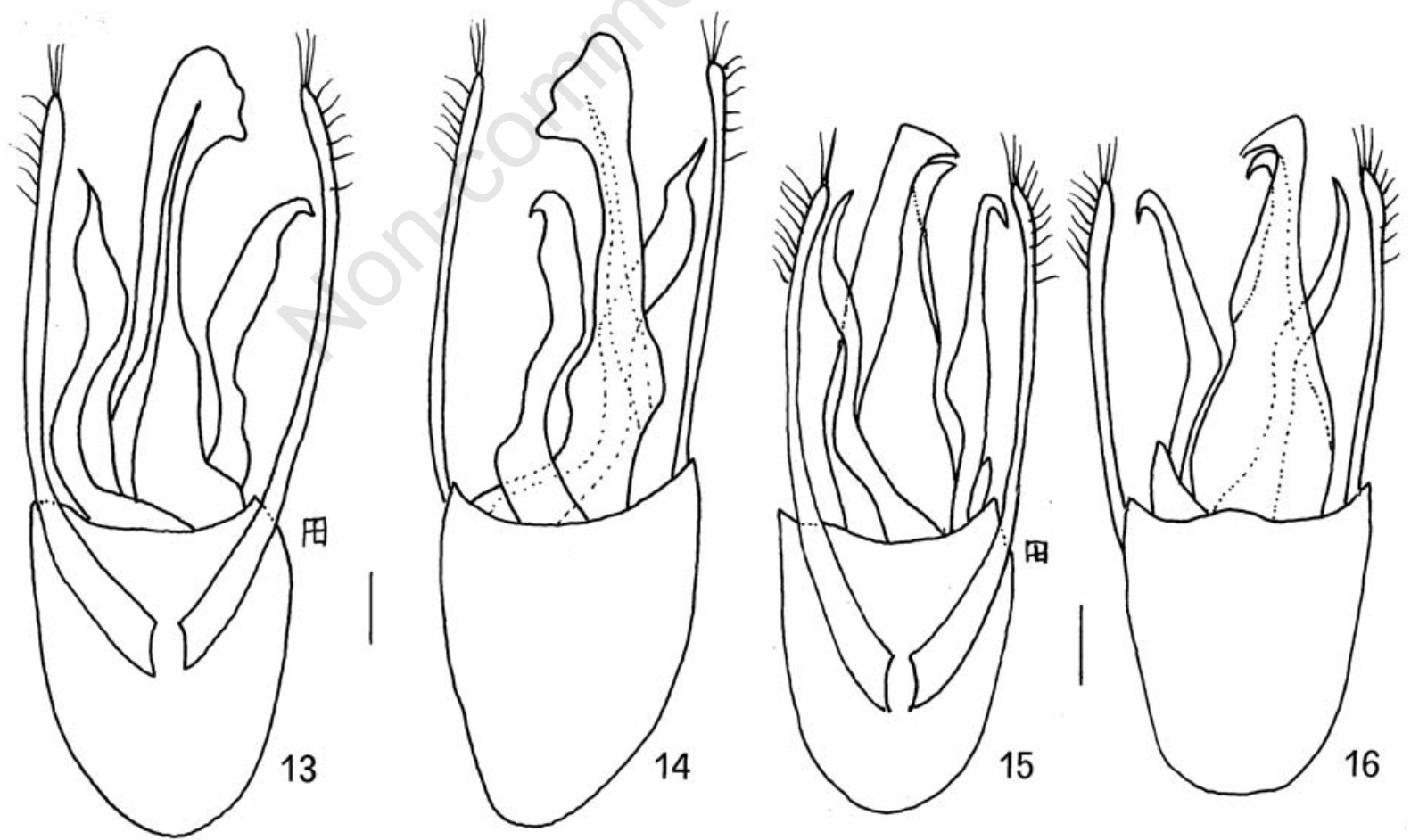

Figg. 13-16. Scotonomus mirificus sp. n.: 13 - edeago in visione ventrale; 14 - in visione dorsale; Scotonomus baroniae sp. n.: 15 - edeago in visione ventrale; 16 - in visione dorsale. Scala: $0.1 \mathrm{~mm}$ 


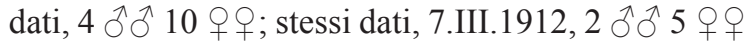
(MSNG, tutti paratipi di S. doderoi Bin.); stessi dati,

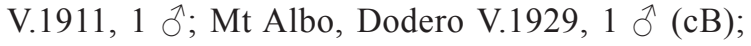
Lula, Mt Albo (Nuoro), Fancello \& Leo leg. 28.XII.1988; stessi dati, 1 수 2 우 (cB).

DesCrizione. Corpo tozzo, più piccolo di quello di $S$. caneparii, lungo $3,5 \mathrm{~mm}$; capo molto dilatato posteriormente, con lati molto largamente arrotondati; pronoto massiccio, molto dilatato in avanti e ristretto posteriormente, con angoli anteriori molto largamente arrotondati; elitre un poco più lunghe della media, a lati ben arrotondati; punteggiatura fine e rada sul capo, fitta sul pronoto; elitre con superficie rugosa.

Edeago (Fig. 19) un poco più corto di $0,8 \mathrm{~mm}$, simile a quello di $S$. caneparii ma con lamella mediana ad apice molto caratteristico, larga alla base e quindi gradatamente ristretta, con dente apicale più tozzo che in S. caneparii; lamella destra, in visione ventrale, spostata verso sinistra, piuttosto robusta ma molto più stretta che in $S$. caneparii, ad apice acuto e non uncinato; lamella sinistra un poco più robusta di quella di S. caneparii, un poco più corta, ad apice acuto e un poco ripiegato; lamella posteriore larga alla base e quindi ristretta, con apice rotondo e caratteristico, come si osserva anche in visione dorsale (Fig. 20).

Distribuzione. Convive sul Monte Albo con $S$. doderoi, S. magrinii, S. mantalbicus sp. n., S. confusus sp. n. e $S$. fauveli sp. n.

Derivatio nominis. La nuova specie prende nome dalla località tipica.

Scotonomus nuragicus Bordoni, 2010 Scotonomus nuragicus Bordoni, 2010: 109.

Materiale tipico. Il tipo ồ è etichettato "Sard. (Nuoro) m 724, Genna Arramene, Consorti leg. 13.III.2007”, Holotypus, Scotonomus nuragicus sp. n., Bordoni det. 2010" (a stampa su cartellino rosso) (cB); paratypi: Baunei (Nuoro), Leo leg.

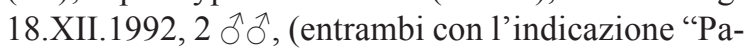
ratypus, Scotonomus nuragicus sp. n., Bordoni det. 2010" a stampa su cartellino rosso) (cB).

Materiale esaminato. Baunei (OG), Chiesa S. Pietro Golgo, 420 m, A. Paladini 9.III.2011, 1 ठै (cP).

Descrizione. Corpo lungo $4,8 \mathrm{~mm}$, simile nell'aspetto generale a $S$. sardus ma con capo meno arrotondato, più dilatato posteriormente, con angoli posteriori più largamente arrotondati, pronoto più massiccio, elitre più larghe.
Edeago (Fig. 21) lungo $1 \mathrm{~mm}$, con lamella mediana fortemente dilatata, ad apice brevemente acuto di lato; lamella destra, in visone ventrale, contorta e stretta, soprattutto nella porzione apicale; lamella sinistra, ancora in visione ventrale, sovrapposta a quella mediana, rivolta verso destra, di forma ondulata, ad apice subacuto; lamella posteriore nascosta dalla lamella mediana, contorta, ad apice uncinato, come si osserva anche in visione dorsale (Fig. 22).

DistRIBUZIONE. La specie è nota del Supramonte meridionale, presso Triei e Baunei, ad ovest del Gennargentu ma presso la costa, a quote non elevate.

Scotonomus doderoi Binaghi, 1970

Scotonomus doderoi Binaghi,1970: 125; Coiffait, 1982: 163 (sub S. raymondi).

Scotonomus troglophilus Coiffait, 1971: 732; 1982: 163, syn. n.

Materiale tipico. Il tipo ô di doderoi è etichettato "Monte Albo, Sardegna, A. Dodero 2.V.1929", "Scotonomus doderoi Binaghi 1970, Holotypus" (su cartellino bianco, di mano di Binaghi) (MSNG); paratypi [tutti con il cartellino arancione "Paratypus (a stampa), Scotonomus doderoi Binaghi 1970" (di mano di Binaghi), "Paratypus" (su cartellino bianco)]: 1 तै 2 우, stessi dati (MSNG); 1 ภै, stessi dati (cB); 1 गे, stessi dati, V.1929; 1 ○े 3 우, stessi dati, 10.III.1912; “Lula, A. Dodero IV.1929”, 2 ふぇ 4 우우; stessi dati, 7.III.1912, 3 ô (MSNG).

Il tipo ô di troglophilus è etichettato "Sicile (sic!), Lula, prés entrée Gr. De Capsa", "Holotype" (a stampa su cartellino rosso), "Scotonomus troglophilus Coiff., H. Coiffait det. 1969” (MNHNP); paratipo [con il cartellino rosso "Paratype" (a stampa) e la medesima determinazione dell'olotipo]: $1 \hat{\jmath}$, stessi dati (MNHNP).

Materiale esaminato. Lula, leg. ? III.1961, 1 ex.; stessi dati, X.1961, 2 exx. (MHNG); Oniferi (Nuoro), M. Curti leg. 6.III.1979, 1 ex. (cB).

DesCrizione. Corpo lungo 3,7-4,2 $\mathrm{mm}$, simile a $S$. binaghii da cui si distingue con difficoltà in base ai caratteri esoscheletrici.

Edeago (Fig. 23) lungo 0,9 mm, con lamella mediana larga, ad apice biforcuto; lamella sinistra, in visione ventrale, accostata alla precedente, molto larga alla base e ristretta nella porzione subapicale, con apice acuto; lamella destra, ancora in visione ventrale, stretta e ad apice subacuto; lamella posteriore 

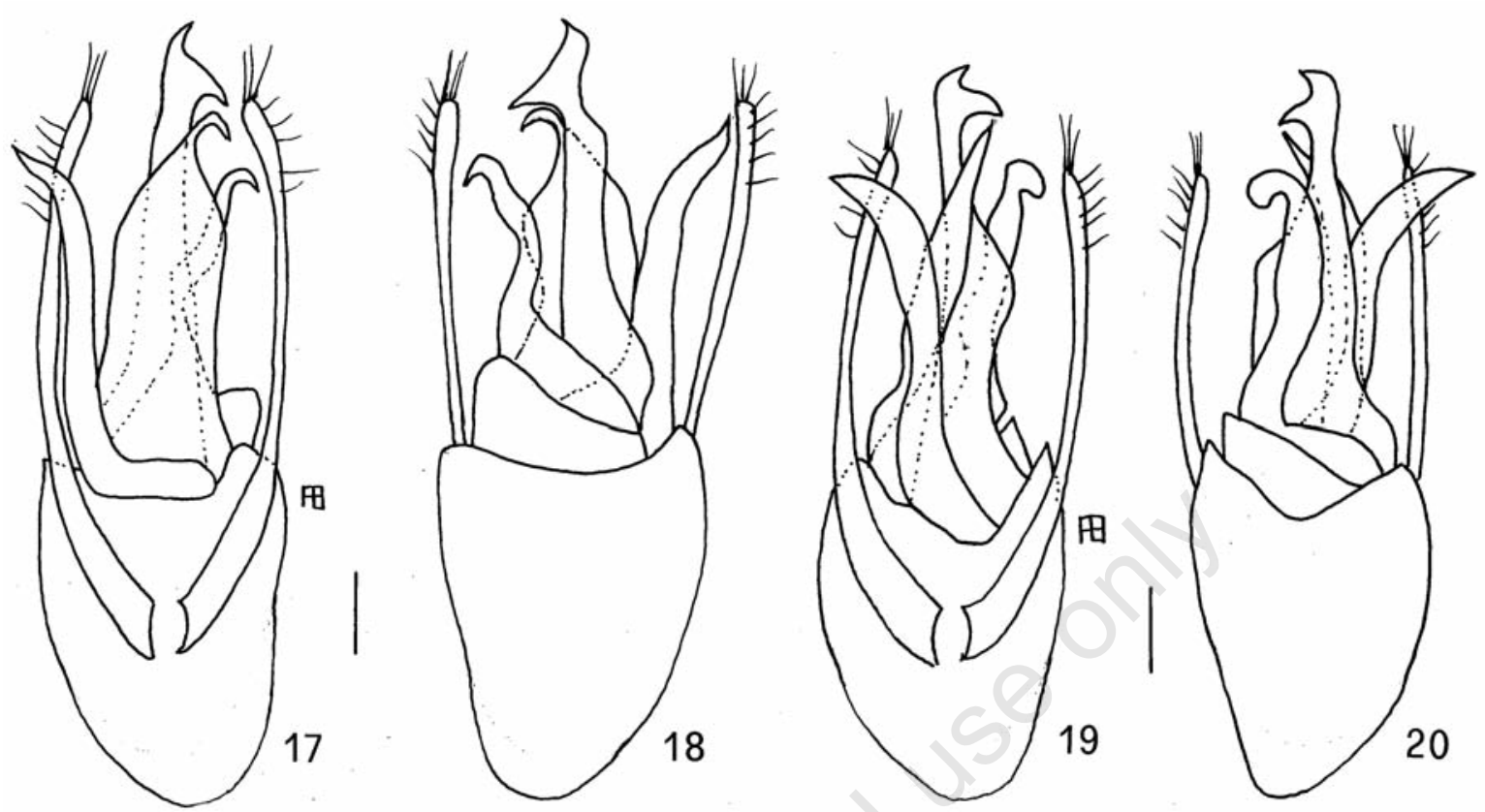

Figg. 17-20. Scotonomus caneparii sp. n.: 17 - edeago in visione ventrale; 18 - in visione dorsale; Scotonomus lulensis sp. n.: 19 - edeago in visione ventrale; 20 - in visione dorsale. Scala: $0.1 \mathrm{~mm}$

larga alla base, quindi ristretta e ad apice uncinato, come si osserva anche in visione dorsale (Fig. 24).

DistribuZione. Monte Albo e dintorni.

Note. Dall'esame di tutti gli esemplari della serie tipica siamo in grado di affermare che sotto il nome $S$. doderoi sono presenti cinque specie distinte: $S$. doderoi, S. binaghii, S. lulensis sp. n., S. confusus sp. n. e $S$. fauveli sp. n.

Binaghi (1970), descrivendo S. doderoi, illustra gli edeagi di due diversi esemplari; le differenze, in realtà piuttosto notevoli, nella conformazione di questi edeagi, vengono attribuite da Binaghi ad una semplice "plasticità....della morfologia delle lamelle". In seguito all'esame dell'holotypus il nome S. doderoi Binaghi, 1970 va attribuito agli esemplari con edeago corrispondente alla Figura 13 del lavoro di Binaghi, mentre gli esemplari con edeago come in Figura 14 dello stesso lavoro sono riferibili a $S$. confusus sp. n. Altri paratipi esaminati sono invece da attribuire a $S$. binaghii Coiffait, $S$. lulensis sp. n., $S$. fauveli sp. $\mathrm{n}$.

Stante la ben nota scrupolosità di Binaghi, appare veramente strano che egli non si sia accorto di ciò, probabilmente anche per non aver effettuato l'estrazione a tutti gli esemplari. Le cinque specie infatti sono mescolate nelle varie brochettes. Trattandosi di specie assai simili, è facile confonderle in base ai soli caratteri esterni, tanto più trattandosi di specie conviventi.

Dall'esame dei tipi di S. troglophilus Coiffait, 1971 (nella cui descrizione non viene ripetuta l'indicazione "Sicile" che è frutto evidentemente di una svista) siamo anche in grado di stabilire la sinonimia di questo con $S$. doderoi Binaghi, 1970.

Successivamente Coiffait (1982) considera $S$. doderoi Binaghi sinonimo di S. raymondi Fvl., senza una motivata giustificazione, tanto più che la figura 13 del contributo di Binaghi, anche se minuta e poco dettagliata, è chiaramente simile a quella, altrettanto approssimativa, del suo troglophilus. In realtà, sulla base dei risultati del presente lavoro, $S$. raymondi Fauvel e $S$. doderoi Binaghi sono da considerarsi specie distinte.

Scotonomus consortii Bordoni \& Magrini, 2003 Scotonomus consortii Bordoni \& Magrini, 2003: 168.

Materiale tipico. Il tipo $\widehat{\partial}$ è etichettato "Sard. 


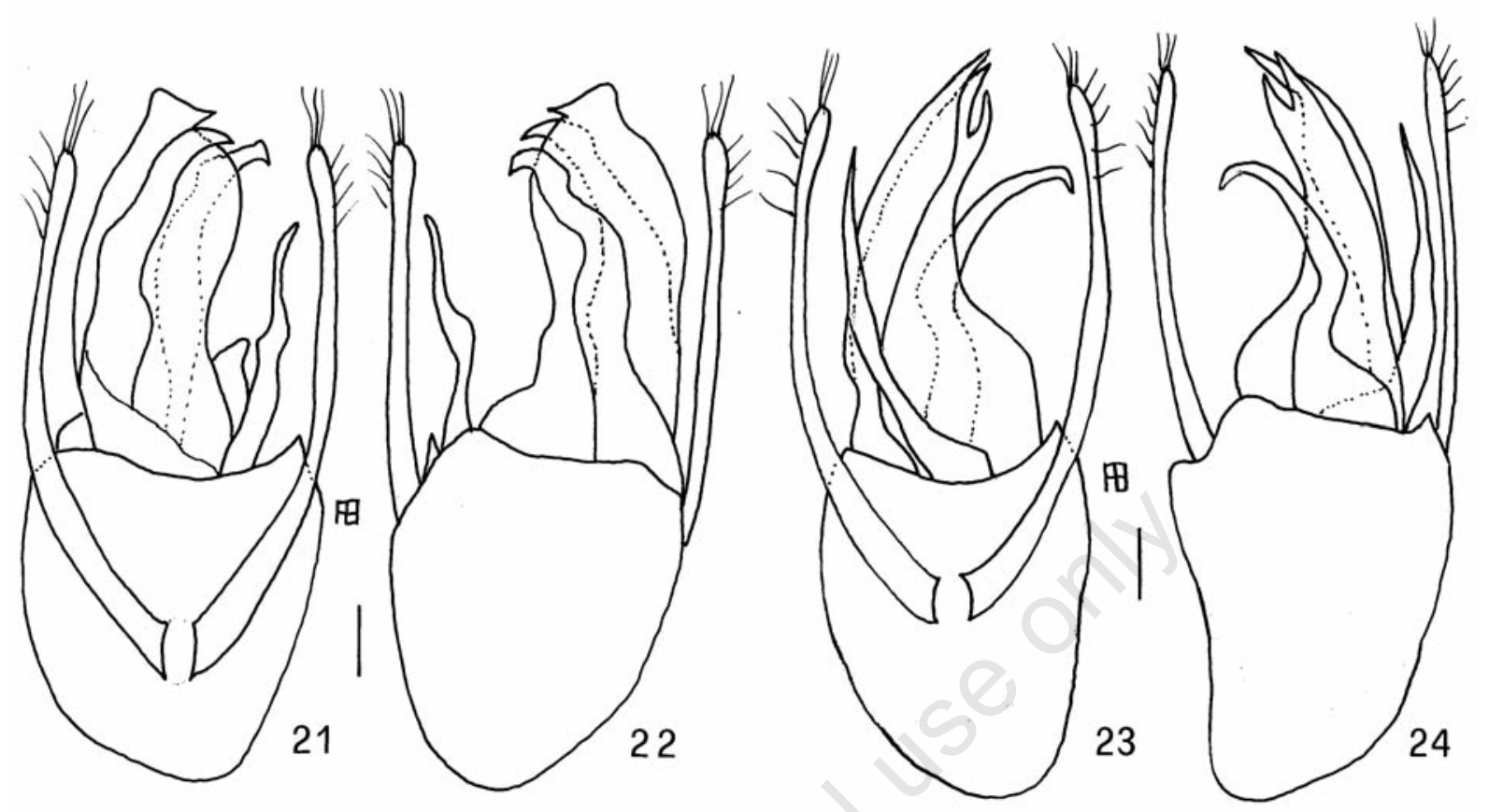

Figg. 21-24. Scotonomus nuragicus Bordoni: 21 - edeago in visione ventrale; 22 - in visione dorsale; Scotonomus doderoi Binaghi: 23 - edeago in visione ventrale; 24 - in visione dorsale. Scala: $0.1 \mathrm{~mm}$

(NU), Mt Tuttavista, Consorti leg. 20.III.2001", "Holotypus, Scotonomus consortii sp. n., Bordoni \& Magrini det. 2002" (a stampa su cartellino rosso) (cB).

Materiale esaminato. Non sono stati reperiti altri esemplari oltre quello tipico.

DESCRIZIONE. Corpo lungo 3,9 mm, affine a binaghii ma con capo a lati meno arrotondati e con punteggiatura più fine, pronoto più corto, con punteggiatura più fine e rada.

Edeago (Fig. 25) piccolo, lungo 0,8 mm, con lamella mediana molto lunga e lungamente ristretta, ad apice vistosamente uncinato; lamella destra, in visione ventrale, sovrapposta alla precedente, per quasi tutta la sua lunghezza ristretta e ad apice acuto; lamella sinistra, ancora in visione ventrale, stretta e ad apice molto acuto; lamella posteriore ben visibile, molto larga alla base quindi ristretta e arcuata nella porzione apicale, con apice acuto, come si osserva anche in visione dorsale (Fig. 26).

Distribuzione. Noto solo della località tipica di Monte Tuttavista, a nord di Dorgali, presso Orosei, a circa $800 \mathrm{~m}$.

\section{Scotonomus magrinii Bordoni, 2006}

Scotonomus magrinii Bordoni, 2006: 8.

Materiale tipico. Il tipo ồ è etichettato "Sardegna, (NU), Siniscola, Mt Albo, Magrini leg. 6.III.2004", "Holotypus, Scotonomus magrinii sp. n., Bordoni det. 2004" (a stampa su cartellino rosso) (cB); paratipi: 2 우우, stessi dati (cM); 1 त 1 우, stessi dati, Consorti leg.; $1 \hat{\jmath}$, stessi dati, Degiovanni leg. (tutti con l'indicazione "Paratypus, Scotonomus magrinii sp. n., Bordoni det. 2004" a stampa su cartellini rossi) (cB).

Materiale esaminato. Lodé (Nuoro), Magrini leg. 7.XI.2005, 1 § (cB).

Descrizione. Corpo lungo $3,5 \mathrm{~mm}$, simile a $S$. binaghii ma più corto e meno slanciato, con capo più dilatato, a lati più largamente arrotondati, con pronoto più largo e lungo, con elitre più corte e dilatate.

Edeago (Fig. 27) piccolo, lungo $0,8 \mathrm{~mm}$, con lamella mediana simile a quella di $S$. consortii ma più larga anche nella porzione preapicale, con apice diversamente conformato; lamella destra, in visione ventrale, vistosamente lunga, rivolta verso la precedente, stretta e ad apice acuto; lamella sinistra, ancora in visione ventrale, sovrapposta alla lamella 


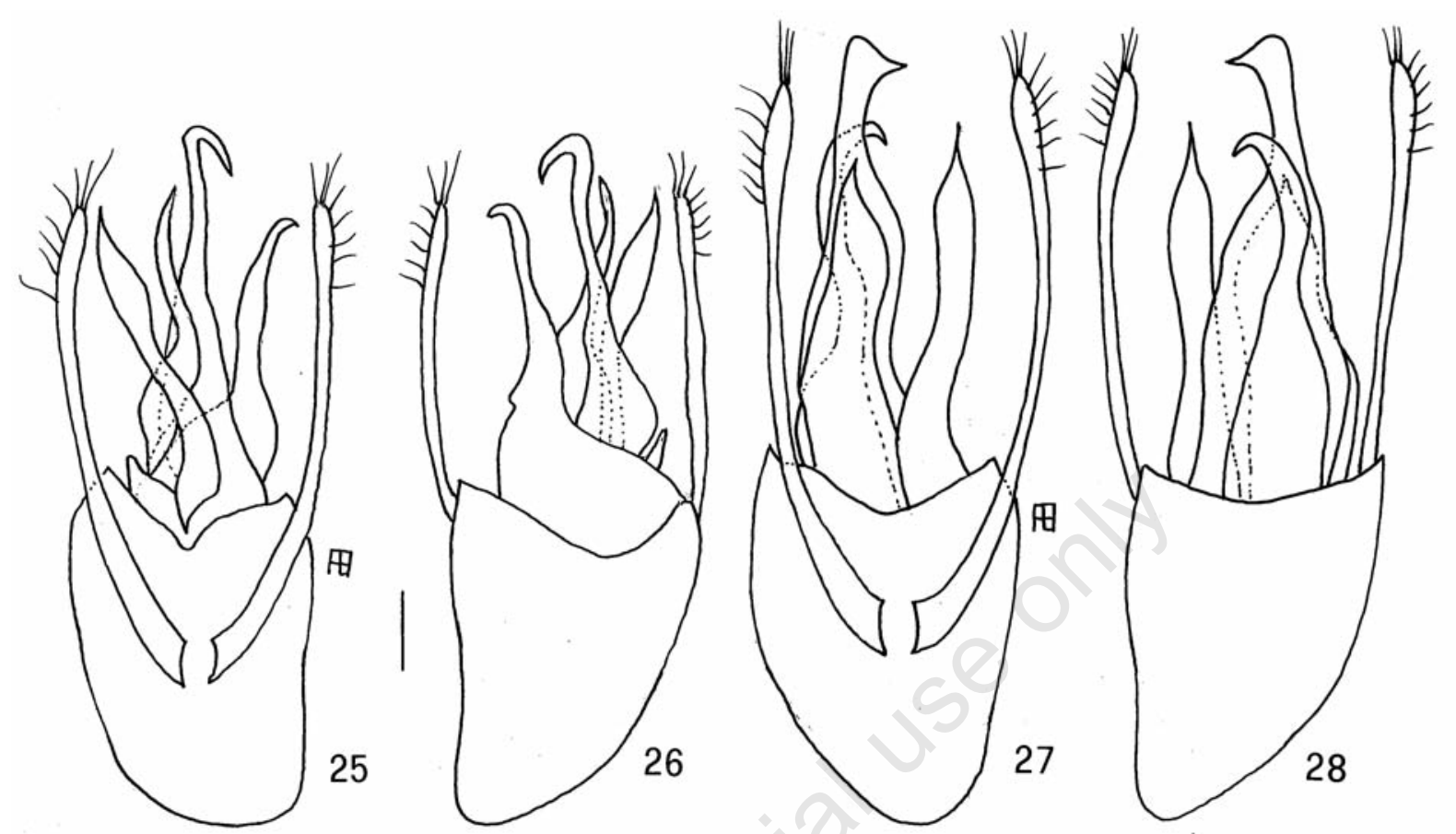

Figg. 25-28. Scotonomus consortii Bordoni \& Magrini: 25 - edeago in visione ventrale; 26 - in visione dorsale; Scotonomus magrinii Bordoni: 27 - edeago in visione ventrale; 28 - in visione dorsale. Scala: $0.1 \mathrm{~mm}$

mediana, ben più corta, ugualmente larga alla base e gradatamente ristretta fino all'apice acuto; lamella posteriore larga alla base, arcuata all'apice, come si osserva anche in visione dorsale (Fig. 28).

DistribuZione. Monte Albo e dintorni.

\section{Scotonomus dorgaliensis sp. $\mathrm{n}$.}

Materiale esaminato. Holotypus $\overbrace{}^{\top}$ : Sardegna, Dorgali (Nuoro), La Traversa, $220 \mathrm{~m}$, Fancello \& Leo leg. 12.V.1991 (cB).

Descrizione. Corpo tozzo, lungo 3,4 $\mathrm{mm}$, simile a quello della specie precedente, con pronoto meno dilatato anteriormente, a lati più vistosamente arrotondati; elitre più corte; punteggiatura di capo ed elitre rada, più fitta sul pronoto.

Edeago (Fig. 29) piccolo, lungo 0,8 mm, con struttura delle lamelle piuttosto complessa. Lamella mediana di forma ondulata, larga nella porzione mediano-basale, quindi ristretta, con apice un poco ripiegato e acuto; lamella destra, in visione ventrale, piuttosto larga e robusta, un poco arcuata, ad apice acuto; lamella sinistra, ancora in visione ventrale, molto larga nella porzione mediano-basale, parzialmente ricoperta dalla lamella sinistra, bruscamente molto ristretta nella porzione apicale che è ripiegata lungamente ad uncino; lamella posteriore larga alla base e quindi ristretta, ad apice uncinato, come si osserva anche in visione dorsale (Fig. 30).

DistribuZione. Noto solo della località tipica.

DERIVATIO NOMINIS. Dal nome della località tipica.

Scotonomus saulcyi Coiffait, 1971

Scotonomus saulcyi Coiffait, 1971: 732; 1982: 162.

Materiale tipico. Il tipo ồ è etichettato "Sardaigne", "Scalla (sic) Cavalli", "Holotype" (a stampa su cartellino rosso), "Scotonomus saulcyi Coiff., H. Coiffait det. 1970" (MHNP).

Materiale esaminato. Padria (Sassari), Fancello \& Leo leg. 16.II.1986, 2 Ђึ 3 우우 (cB); stessi dati, Leo leg. 16.XII.1986, 1 त ; stessi dati, Fancello leg. 16.XII.1986, 1 ๆ (cC); stessi dati, 3 के के (cB). 
DESCRIZIONE. Corpo minuto, lungo 3,2 mm; capo subrettangolare, non dilatato posteriormente, appena ristretto in avanti, con lati subrettilinei; pronoto non massiccio, dilatato anteriormente e molto ristretto posteriormente; elitre non particolarmente corte; punteggiatura più fine della media.

Edeago (Fig. 31) molto piccolo, lungo 0,7 $\mathrm{mm}$, con lamella mediana molto stretta rispetto alla norma, ad apice ricurvo e acuto; lamella destra, in visione ventrale, stretta e dritta, ad apice acuto; lamella sinistra, ancora in visione ventrale, accostata alla lamella mediana, un poco più corta e più o meno della medesima forma; lamella posteriore stretta, piegata un poco a gomito a circa metà della sua lunghezza, con apice uncinato, come si osserva anche in visione dorsale (Fig. 32).

DistribuZIONE. Sassarese orientale.

Note. Attribuiamo a S. saulcyi gli esemplari raccolti nella stazione di Padria (località assai prossima al locus typicus: Cantoniera di Scala Cavalli presso Uri, cfr Bordoni, 2006) in quanto ben corrispondenti come morfologia esterna ed edeagica all'holotypus immaturo da noi esaminato, benchè l'edeago di quest'ultimo si presenti, nel preparato microscopico, poco chitinizzato e diafano.

Coiffait riporta la località di "Scala Caval" non avendo evidentemente notato che di seguito sul cartellino, a capo, vi è scritto "li".

Il tipo di questa specie è stato fortunosamente rintracciato, non si sa per quale motivo, nella collezione dell'entomologo francese André Argod-Villon (1859-1936), anziché in quella Saulcy, come indicato dal suo autore.

\section{Scotonomus raymondi Fauvel, 1873}

Scotonomus raymondi Fauvel, 1873: 328; Coiffait, 1971: 730; 1982: 163.

MATERIALE TIPICO. In questa sede consideriamo come typus un esemplare $\delta^{\lambda}$ etichettato "Sardaigne, Raymond", "Raymondi Fauv." (di mano di Fauvel), "Coll. et det A. Fauvel, Scotonomus etruscus Saulcy, R. I. Sc. N. B. 17479”, “G. Fagel 1962, Scotonomus Raymondi Fauv.", "Lectotype Coiffait 1970" (a mano su cartellino rosso) (IRSNB).

MATERIALE esAminato. I soli altri esemplari esaminati, oltre al typus succitato, sono $4 \hat{\delta} \hat{\delta}$ etichettati l'uno "Sardaigne", "Scotonomus raymondi Fvl., H. Coiffait det. 1970", "comparé au type ${ }^{\lambda "}$ (su cartel- lino rosso) (MHNP), l'altro "Sardinia" (cB) ed il terzo "Sard., Sassari, 1869" (cB), il quarto "Ambros., Cors.", riferibile ai dintorni di Sassari (cfr. Note).

DesCrizione. Corpo lungo 3,6 mm; capo un poco dilatato posteriormente, con lati molto arrotondati; pronoto non particolarmente massiccio ma ben dilatato in avanti e ristretto posteriormente, con lati subrettilinei; elitre molto larghe, corte, ad omeri largamente arrotondati; punteggiatura di capo e pronoto piuttosto grossa; elitre con superficie rugosa.

Edeago (Fig. 33) lungo $1 \mathrm{~mm}$, con lamella mediana molto lunga, spessa, gradatamente ristretta verso la porzione apicale, con apice a becco; lamella destra, in visione ventrale, stretta e molto corta, più corta che in tutte le altre specie sarde; lamella sinistra, ancora in visione ventrale, gradatamente ristretta verso l'apice che è acuto; lamella posteriore più o meno a forma di $\mathrm{S}$, stretta, con apice uncinato, come si osserva anche in visione dorsale (Fig. 34).

Distribuzione. Sembrerebbe che la specie, assai sporadica, abiti nella Sardegna settentrionale, nel Sassarese.

Note. L'esemplare di S. raymondi distinto da Coiffait con il cartellino di Lectotypus, è stato rintracciato (IRSNB), in seguito ad accurate ricerche, mescolato ad una piccola serie di S. etruscus Saulcy, 1878, e determinato come tale; le figure $30 \mathrm{~A}-\mathrm{B}-\mathrm{C}$ in Coiffait (1982) si riferiscono ad un esemplare di Ozieri (Sassari), località che non è riportata sul cartellino di questo esemplare, indicato da Coiffait come Lectotypus, per cui si può supporre che l'autore francese abbia esaminato almeno un altro esemplare etichettato "Ozieri" di cui tuttavia non vi è traccia. Non risulta che sia stata pubblicata da Coiffait la designazione a lectotipo dell'esemplare conservato all'IRSNB, per cui in questa sede viene scelto comunque questo esemplare (etichettato "Sardaigne, Raymond", "Raymondi Fauv." (di mano di Fauvel), "Coll. et det A. Fauvel, Scotonomus etruscus Saulcy, R. I. Sc. N. B. 17479", “G. Fagel 1962, Scotonomus Raymondi Fauv."), designandolo ex novo come lectotypus della specie.

Le citazioni relative a $S$ raymondi in Binaghi (1970) e Bordoni \& Magrini (2003) sono in realtà da riferire a $S$. binaghii Coiffait.

Riguardo al restante materiale da noi esaminato e riferibile a $S$. raymondi, l'esemplare con cartellino di Coiffait "comparé au type ${ }^{\lambda}$ ", è stato 

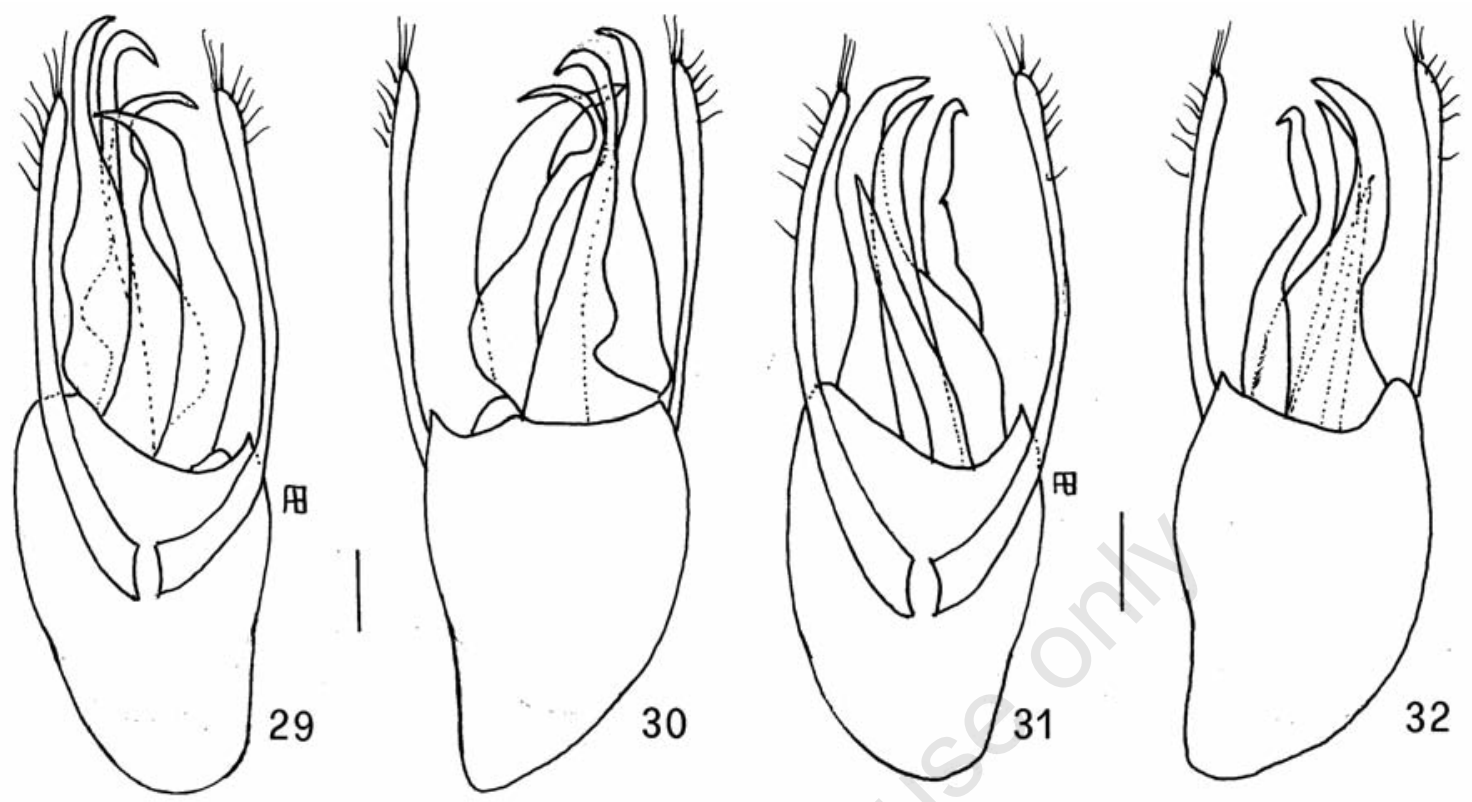

Figg. 29-32. Scotonomus dorgaliensis sp. n.: 29 - edeago in visione ventrale; 30 - in visione dorsale; Scotonomus saulcyi Coiffait: 31 - edeago in visione ventrale; 32 - in visione dorsale. Scala: $0.1 \mathrm{~mm}$

rintracciato nella collezione Argod-Vallon (MNHNP), assieme al typus di S. saulcyi.

Nelle collezioni del Deutsches Entomologisches Institut è conservato un esemplare etichettato "Sardinia", "coll. Stierlin", "S. Raymondi Saulcy (sic), Raymond" (a mano), "histor Exempl., vielleicht Type" (a stampa su cartellino rosso): si tratta di una femmina, quindi impossibile da identificare con certezza

Nelle collezioni del Naturhistorisches Museum di Vienna è conservato un maschio etichettato "Ambros. Cors." (ovvero Ambrosini leg., Corsica): si tratta evidentemente di una errata cartellinatura riferibile, in base ad analoghi casi, ai dintorni di Sassari (Poggi, com. pers.).

Con questo contributo la sinonimia tra S. raymondi e $S$. doderoi viene smentita.

\section{Scotonomus confusus sp. n.}

Materiale esaminato. Holotypus $\widehat{\jmath}$ : Sardegna, Lula (Nuoro), Dodero VI.1911 (MSNG, paratipo di S. do-

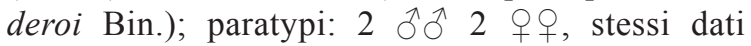
(MSNG); 1 ${ }^{\Uparrow} 1$ ㅇ stessi dati (cB); Mt Albo (Nuoro), Dodero 10.III.1912, 1 đ̃; Dorgali (Nuoro), Dodero
V.1910, 1 \ 2 우 (MSNG, tutti paratipi di S. doderoi Bin.); Dorgali (Nuoro), Mt Coazza, Fancello \& Leo leg. 12.V.1991, 2 đै (cB); Grotta Pisanu, Dorgali (NU), P. Magrini leg. 19.VIII.2012, 1 ठ̊ (cB).

DESCRIZIONE. Corpo lungo 3,5 mm; capo moderatamente dilatato in addietro, con lati ben arrotondati; pronoto dilatato anteriormente e molto ristretto in addietro, con lati ben arrotondati; elitre corte, con omeri molto arrotondati; punteggiatura piuttosto grossa su capo e pronoto, molto fine sulle elitre.

Edeago (Fig. 35) piuttosto piccolo, lungo 0,8 $\mathrm{mm}$, con lamella mediana robusta, gradatamente ristretta, ad apice ricurvo e acuto; lamella destra, in visione ventrale, simile alla precedente alla quale è accostata, quasi della medesima lunghezza, ad apice acuto; lamella sinistra, ancora in visione ventrale, un poco arcuata, ad apice acuto; lamella posteriore sinuosa, ristretta nella porzione apicale, con apice vistosamente uncinato, come si osserva anche in visione dorsale (Fig. 36).

Distribuzione. Nota del Monte Albo e dintorni e di Dorgali.

DERIVATIO NOMINIS. Il nome confusus deriva dal fatto 

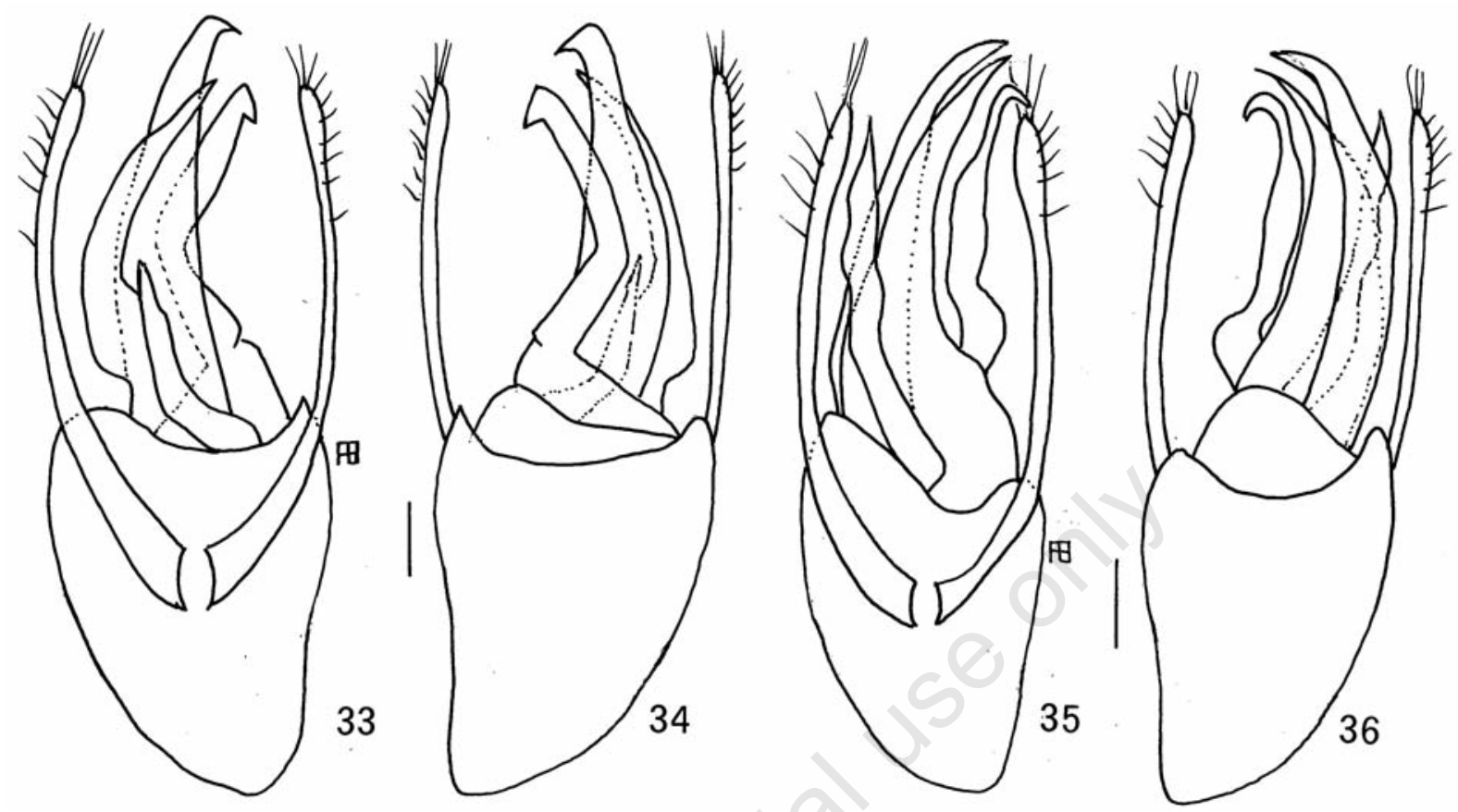

Figg. 33-36. Scotonomus raymondi Fauvel: 33 - edeago in visione ventrale; 34 - in visione dorsale; Scotonomus confusus sp. n.: 35 - edeago in visione ventrale; 36 - in visione dorsale. Scala: $0.1 \mathrm{~mm}$

che alcuni esemplari della nuova specie erano frammisti nella serie tipica di $S$. doderoi.

Scotonomus montalbicus $\mathrm{sp}$. n.

Materiale esaminato. Holotypus $\delta$ : Sardegna, Lula (Nuoro), Monte Albo, Fancello \& Leo leg. 11.III.1989 (cB); paratypi: stessi dati, 3 ふึત 2 우우 (cB).

DesCrizIOne. Corpo lungo circa $4 \mathrm{~mm}$; capo largo e corto, molto dilatato posteriormente, a lati molto largamente arrotondati; pronoto proporzionalmente corto, dilatato in avanti, a lati appena arrotondati; elitre simili a quelle della specie precedente; punteggiatura del pronoto regolare e fitta.

Edeago (Fig. 37) lungo 0,9 mm, con lamella mediana molto spessa, massiccia, ad apice ricurvo ed acuto; lamella destra, in visione ventrale, molto grande, ben più lunga delle altre lamelle, posta dietro quella mediana, ad apice ricurvo; lamella sinistra, ancora in visione ventrale, molto lunga e stretta, più o meno a forma di S, ad apice acuto; lamella posteriore stretta, un poco ripiegata a gomito a circa metà della sua lunghezza, ad apice nettamente uncinato, come si osserva anche in visione dorsale (Fig. 38).

Distribuzione. Nota solo del Monte Albo.

DERIVATIO NOMINIS. La nuova specie prende nome dall'imponente massiccio calcareo in cui vive.

\section{Scotonomus binaghii Coiffait, 1982}

Scotonomus binaghii Coiffait, 1982: 163.

Scotonomus doderoi Coiffait, 1971: 732.

Materiale TIPICO. Il tipo di questa specie è un $\widehat{\partial}$ etichettato "Macomer (Sard.), A. Dodero 5.XI.1909", "Holotype" (a stampa su cartellino rosso), "Scotonomus doderoi Coiff., H. Coiffait det. 1970" (MHNP).

Materiale esaminato. Ozieri (Sassari ), Dodero

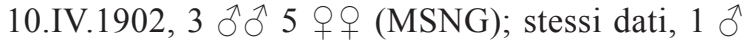
(IRSNB); Ozieri (Sassari), Dodero leg., $1 \lambda$ (MNHU); stessi dati, Sbordoni leg. 29.IV.1987, 1 ठ (cB), 1 ㅇ (cT); Ozieri (Sassari), A. Dodero leg., 1 ๙ 1 q (DEI); Ozieri, A. Dodero 23.XII.1884, 1 ex.; A. Dodero 20.XI.1892, 4 exx. (NMW); Ozieri, 1 o (MSNM); Ozieri, 3 exx. (MHNG); Macomer 
(Nuoro), Dodero leg. 27.IV.1908, 1 \ 2 우; stessi dati, senza data, 1 ઈิ 1 ㅇ (MSNG); Macomer (Nuoro), A. Dodero leg. Febbr. 1892, 1 (MNHU); stessi dati, Fancello leg. 12.IV.1995, 1 गे; stessi dati 22.XI.1990, 1 q; stessi dati 10.III.1992, 1 ô; stessi dati, 12.X.1990, 1 § (cC); Borore (Nuoro), Sbordoni leg. 30.IV.1967, 1 ते (cB); stessi dati, Leo leg. 15.XII.1985, 2 $\widehat{\delta}$ (cL); Macomer (Nuoro), Mt S. Antonio, Dodero leg. 9.X.1909, 1 으 2 우 (MSNG); stessi dati, Sbordoni \& Vigna leg. 2.V.1967, 1ठ̄; stessi dati, Sbordoni leg. 8.V.1967, 1 đ; stessi dati, 30.IV.1967, 1 ○ (cB), 1 ex. (MHNG); stessi dati, Fancello \& Leo leg. 26.II.1986, 1 주 2 우 (cB); stessi dati, senza data, 1 + (cC); Macomer (Nuoro), Stagno di Bara (NU), Fancello leg. 20.II.1986, 1 ㅇ (cC); stessi dati, Fancello \& Leo leg. 20.III.1987, 1 고 4 우우 (cB); stessi dati, Magrini leg. III.2004, 1 수 (cB); Oniferi, Rio Sas Coronas (Nuoro), Fancello \& Leo leg. 12.V.1991, 1 ○ 1 ๆ (cB); Dualchi (Nuoro), Fancello \& Leo leg. 16.II.1986, 2 + $O$; stessi dati, 8.XII.1985, 1 ô; stessi dati, Leo leg. 20.III.1987, 1

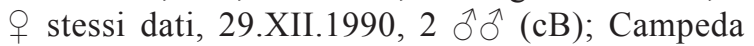
(Nuoro), Dodero leg. 24.XI.1903, 2 추 3 우우 (MSNG), 1 ex. (MCZ); Oliena, Su Gologone, P. Henrot leg. 9.IV.1956, 2 exx. (MZUR); Mt Ferru (Oristano), Dodero leg. 2.V.1891, 1 q; stessi dati, senza data, 1 수 4 우 (MSNG); Bauladu (Oristano), Fancello \& Leo leg. 1.III.1989, 2 우; stessi dati, 12.V.1991, 2 ठิ ठิ 3 우; stessi dati, Leo leg. 17.XI.1985, 1 ㅇ (cB); Mt Arci (Oristano), Fancello leg. 5.III.1986, 1 §’; stessi dati, 10.XII.1992, 1 우 (cB); stessi dati, 10.12.1992, 1 \% (cC); stessi dati, Fancello \& Leo leg. 27.XII.1989, 3 ô 0 1 1 ㅇ (cB); 1 $\widehat{\partial}$ senza cartellino di località; $2 \hat{\delta} \widehat{\partial}$, Sardaigne (entrambi con la doppia determinazione $S$. raymondi e S. etruscus); 1 ô, Sardinia; 1 Oे, Sardinia, Lostia (entrambi con la doppia determinazione $S$. doderoi e $S$.

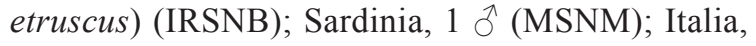
Merkl, 1 ex. (NMW)

DESCRIZIONE. Corpo lungo 4-4,2 mm; capo un poco dilatato posteriormente, con lati un poco arrotondati; pronoto proporzionalmente corto, massiccio, dilatato anteriormente, con lati poco arrotondati; elitre corte; punteggiatura piuttosto grossa e piuttosto fitta.

Edeago (Fig. 39) lungo 0,9 mm, con lamella mediana lunga, ben più delle altre lamelle, larga alla base e per lungo tratto ristretta nella porzione distale, bruscamente ripiegata a gomito nel terzo prossimale, con apice a becco; lamella destra, in visione ventrale, molto robusta, spostata al centro, con apice molto ricurvo e acuto; lamella sinistra, ancora in visione ventrale, della larghezza di quella mediana nella sua porzione mediano-apicale, con apice ricurvo e acuto; lamella posteriore molto larga alla base, quindi bruscamente ristretta, con apice uncinato, come si osserva anche in visione dorsale (Fig. 40).

Distribuzione. E' questa la specie più diffusa in Sardegna, nella porzione centro-occidentale dell'isola. Nella porzione centro-orientale vive la specie successiva, che le è affine.

Note. Coiffait (1982) diede il nuovo nome di S. binaghii al suo $S$. doderoi descritto nel 1971, omonimo juniore di S. doderoi Binaghi, 1970. In effetti Binaghi (1970) riferiva gli esemplari con edeago corrispondente alla figura 15 del suo lavoro a $S$. raymondi mentre essi vanno invece riferiti alla specie descritta da Coiffait come $S$. doderoi, nome poi mutato in $S$. binaghii.

S. binaghii appare affine a $S$. fauveli sp. n. (descritta di seguito), dalla quale differisce sostanzialmente per la conformazione della lamella mediana e di quella posteriore, la prima ristretta per un lungo tratto mediano-prossimale e piegata a gomito, mentre in $S$. fauveli sp. n. è molto più dilatata e ristretta solo nella porzione apicale; la seconda ad apice più stretto ed acuto mentre in $S$. fauveli è più spessa.

\section{Scotonomus fauveli $\mathrm{sp} . \mathrm{n}$.}

Materiale esaminato. Holotypus $\widehat{T}$ : Sardegna, Dorgali (Nuoro), Mt Coazza, Fancello \& Leo leg. 12.V.1991 (cB); paratypi: stessi dati, 1 ते; Nuoro (Nuoro), La Solitudine, Fancello leg. 14.IV.2010, 10; Burgos (Sassari), Foresta di Burgos, Fancello \& Leo leg. 19.XII.1989, 2 ふ઼ (cB); Mt Albo (Nuoro), Dodero leg. II.1929, 1 đ̊; Lula (Nuoro), Dodero leg.

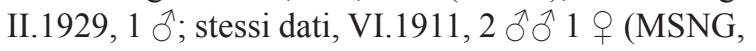
paratipi di S. doderoi Binaghi); Sardinia, Cagliari, Reitter, 1 ô (NMW); Cagliari, U. Lostia, 1 ô (cB);

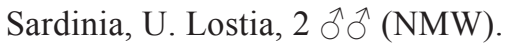

Descrizione. Corpo lungo 3,5-3,7 mm, simile alla specie precedente ma chiaramente più piccolo, con capo più stretto e in avanti più ristretto; pronoto ad angoli anteriori più largamente arrotondati; elitre più strette; punteggiatura simile, un poco meno fitta.

Edeago (Fig. 41) lungo $0,8 \mathrm{~mm}$, un poco più corto di quello della specie precedente, con lamella 

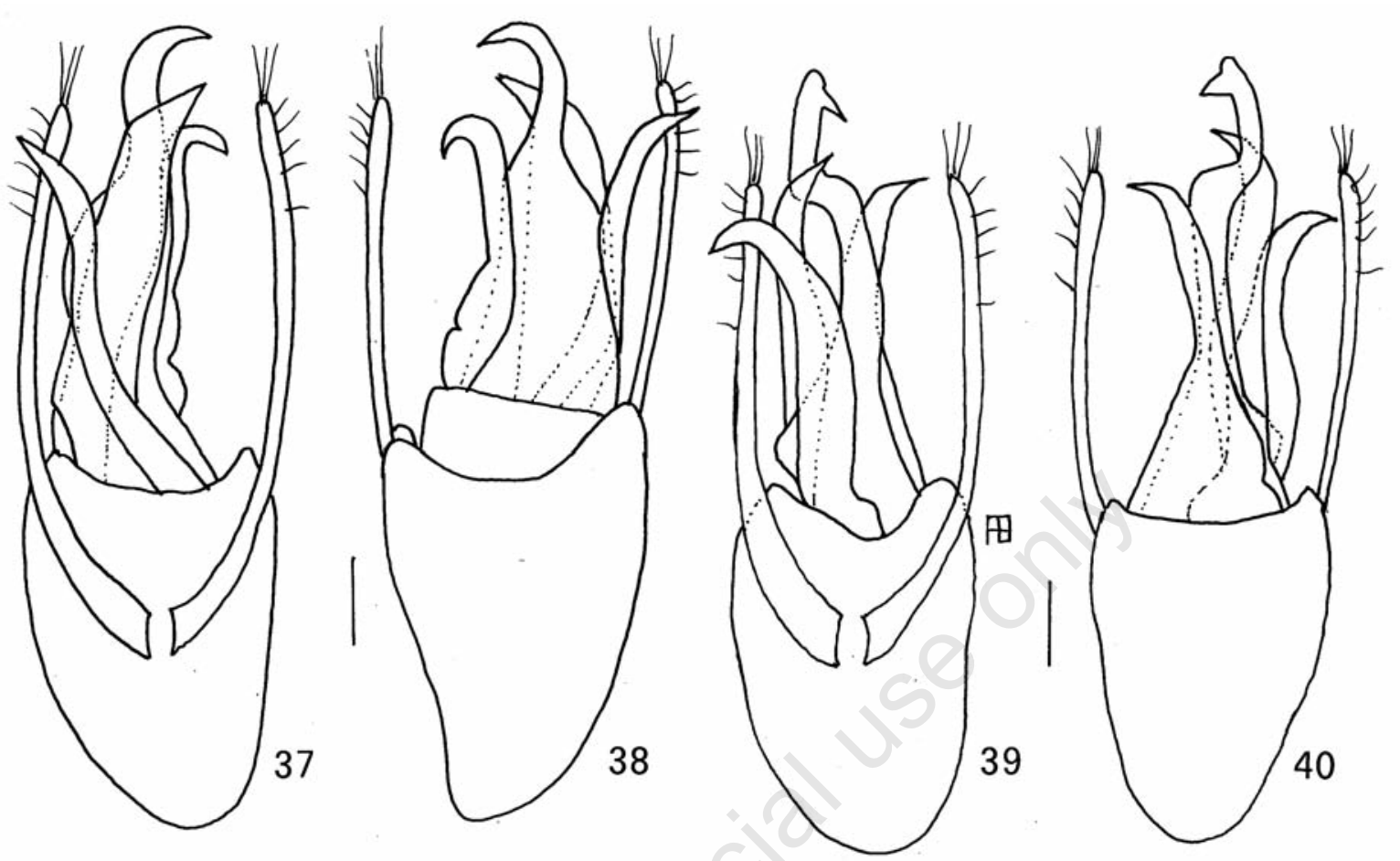

Figg. 37-40. Scotonomus mantalbicus sp. n.: 37 - edeago in visione ventrale; 38 - in visione dorsale; Scotonomus binaghii Coiffait: 39 - edeago in visione ventrale; 40 - in visione dorsale. Scala: $0.1 \mathrm{~mm}$

mediana molto più larga e più corta che in S. binaghii, ristretta solo nella porzione apicale, non piegata a gomito, con apice simile; lamella destra, in visione ventrale, assai simile a quella di S. binaghii ma ad apice meno ricurvo; lamella sinistra, ancora in visione ventrale, simile a quella di S. binaghii ma più robusta; lamella posteriore più stretta alla base, con apice molto più largo e uncinato, come si osserva anche in visione dorsale (Fig. 42).

DistRIBUZIONE. Occupa la porzione centro-orientale della Sardegna ma con una stazione un poco disgiunta nella regione del Goceano (Foresta di Burgos) ed una meridionale nel Cagliaritano. Al Monte Coazza convive con $S$. confusus sp. n.

Derivatio nOMinis. La nuova specie è dedicata a Charles A. Albert Fauvel, descrittore del genere Scotonomus.

Note. Oltre agli esemplari della serie tipica, abbiamo

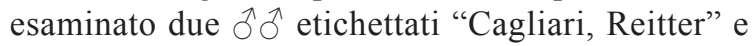
"Cagliari, Lostia" (NMW, cB): a nostro avviso non può trattarsi che di un errore di località.

\section{CONCLUSIONI}

Gli Scotonomus sardi (Fig. 46) possono essere suddivisi in alcuni gruppi di specie in base ai caratteri dell'edeago che, come già indicato, sono gli unici che ne consentono l'identificazione sicura.

Nel gruppo sardus sono incluse le specie che hanno edeago con lamella mediana larga, provvista di un dente vistoso al lato sinistro, con l'organo in visione ventrale. A questo gruppo vengono riferite le seguenti specie: S. sardus Fauvel., S. terteniensis sp. n., S. bifidus sp. n., S. kraussei sp. n. e $S$. barbaricinus sp. n. (Fig. 43).

Nel gruppo simpatricus sono incluse le specie che presentano lamella mediana lunga, più o meno stretta, con apice più o meno dilatato, mai ristretto $\mathrm{e}$ quindi non subacuto, acuto e più o meno ricurvo. A questo gruppo vengono riferite le seguenti specie: $S$. simpatricus Binaghi, S. mirificus sp. n., S. baroniae sp. n., S. caneparii sp. n., S. lulensis sp. n., S. nuragicus Bordoni e $S$. doderoi Binaghi (Fig. 44).

Nel gruppo raymondi sono incluse le specie che 

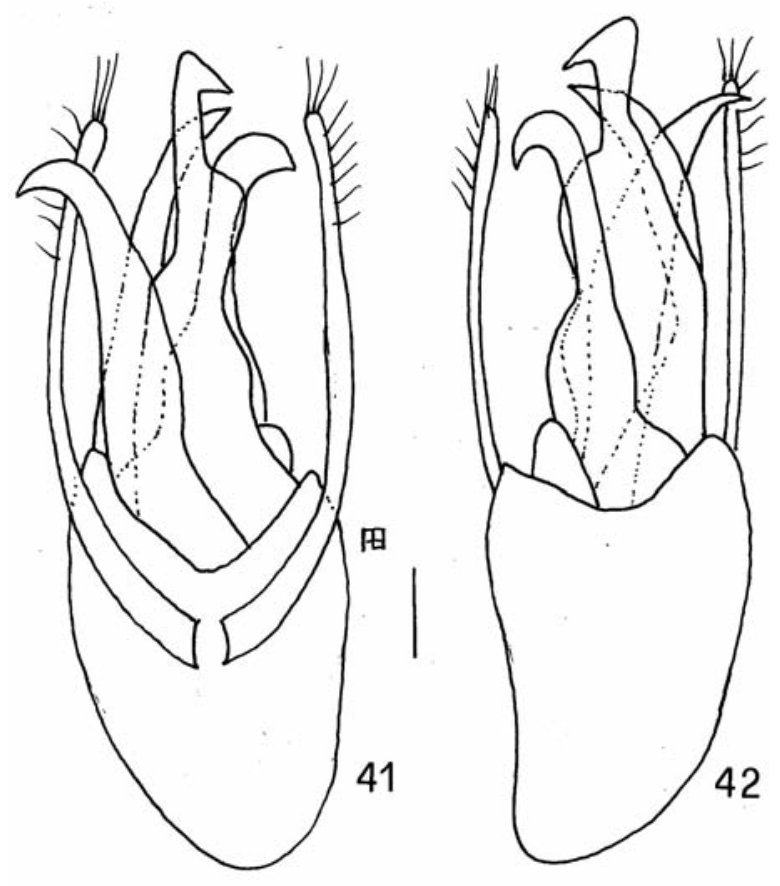

Fig. 41-42. Scotonomus fauveli sp. n.: 41 - edeago in visione ventrale; 42 - in visione dorsale. Scala: $0.1 \mathrm{~mm}$

hanno lamella mediana prevalentemente stretta per lungo tratto, ad apice più o meno acuto e spesso ricurvo o uncinato. A questo gruppo vengono riferite le seguenti specie: S. consortii Bordoni \& Magrini, S. magrinii Bordoni, S. dorgaliensis sp. n., S. saulcyi Coiffait, $S$. raymondi Fauvel., S. confusus sp. n., S. montalbicus sp. n., S. binaghii Coiffait e $S$. fauveli sp. n. (Fig. 45).

\section{Chiave dicotomica Delle SPECIE}

1. Edeago con lamella mediana provvista di un dente molto evidente (gruppo sardus)

-. Edeago con lamella mediana lunga, con apice più o meno dilatato e non ricurvo (gruppo simpatricus)

-. Edeago con lamella mediana ad apice più o meno acuto e spesso ricurvo o uncinato (gruppo raymondi)

\section{...4}

2. Lamella mediana ad apice acuto, con un lungo dente apicale (Fig. 1); lamella posteriore appena arcuata all'apice (Fig. 2); è la specie più robusta in Sardegna $(4,5-5,1 \mathrm{~mm})$ S. sardus Fagel, 1969
-. Lamella mediana ad apice acuto, con un dente apicale molto corto (Fig. 3); lamella posteriore uncinata (Fig. 4)

S. terteniensis sp. n.

-. Lamella mediana ad apice acuto, con dente apicale biforcuto (Fig. 5)

S. bifidus sp. n.

-. Lamella mediana da apice tronco, con un dente biforcuto (Fig. 7); lamella destra molto lunga ....

..S. kraussei sp. n.

-. Lamella mediana ad apice tronco, con un dente preapicale (Fig. 9); lamella destra molto corta (Fig. 10)...

S. barbaricinus sp. n.

3. Lamella mediana larga, ad apice caratteristico (Fig. 19), con lamella destra stretta, corta, ad apice tondo (Fig. 12).

S. simpatricus Binaghi 1970

-. Lamella mediana molto lunga, ad apice largamente arrotondato (Fig. 13), con lamella destra spessa e ad apice uncinato (Fig. 14).

.S. mirificus sp. n.

-. Lamella mediana molto lunga, ad apice stretto ed uncinato /Fig. 15); lamella destra stretta e ad apice uncinato (Fig. 16)

S. baroniae sp. n.

-. Lamella mediana stretta, ad apice di forma caratteristica (Fig. 17), con lamella destra molto larga, sovrapposta alla precedente, ristretta all'apice che è uncinato; lamella posteriore sinuosa e ad apice uncinato (Fig. 18) S. caneparii sp. $\mathrm{n}$.

-. Lamella mediana più larga nella porzione mediano-basale, ad apice simile a quello della specie precedente ma più tozzo, con lamella destra stretta, sovrapposta alla precedente, gradatamente ristretta verso l'apice acuto; lamella posteriore più robusta e ad apice largamente arrotondato (Fig. 20)

S. lulensis sp. n.

-. Lamella mediana molto larga, fortemente dilatata verso destra (Fig. 21)

S. nuragicus Bordoni, 2010

-. Lamella mediana larga, ad apice biforcuto (Fig. 23)

.S. doderoi Binaghi, 1970

4. Lamella mediana sottile nella porzione medianoapicale, ad apice fortemente uncinato (Fig. 25); lamella destra sovrapposta a quella precedente, lungamente ristretta nella porzione mediano-apicale, con apice acuto; lamella posteriore a base molto larga e ad apice brevemente uncinato (Fig. 26) ..........S. consortii Bordoni \& Magrini, 2003

-. Lamella mediana larga, ristretta verso l'apice che ha una sporgenza dentiforme laterale (Fig. 27); lamella destra vistosamente lunga, rivolta verso 


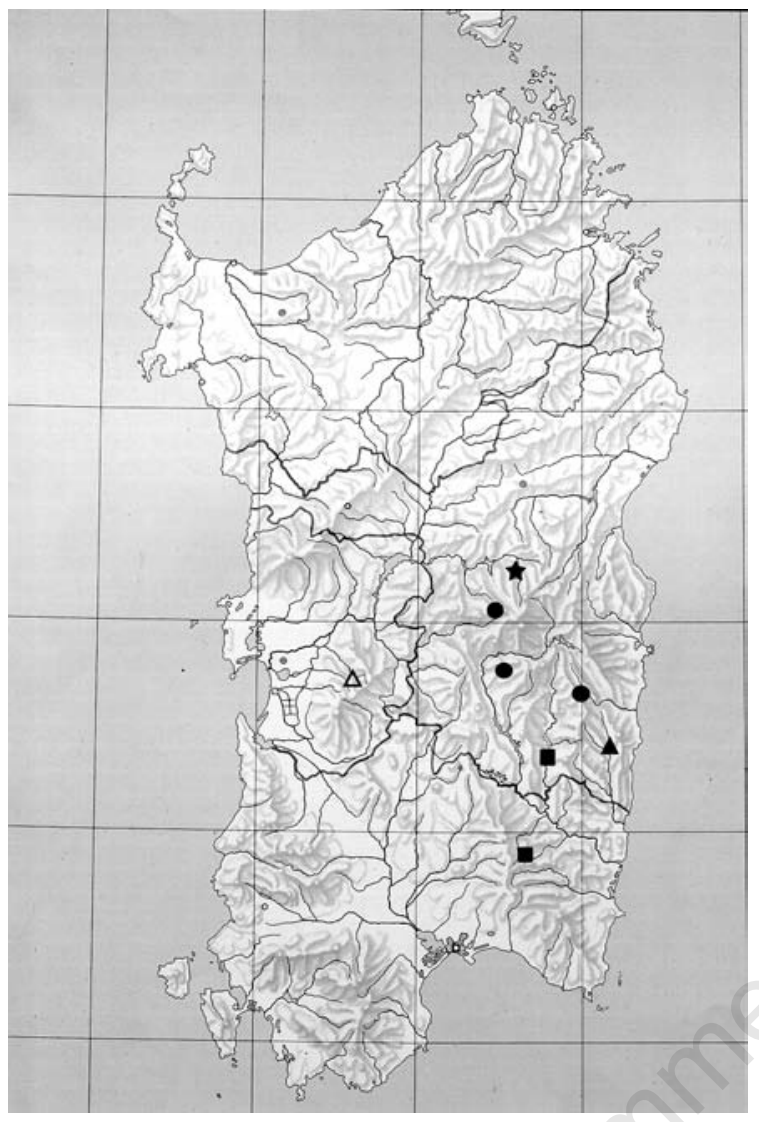

Fig. 43. Distribuzione degli Scotonomus del gruppo sardus: $S$. sardus Fagel (tondo), S. terteniensis sp. n. (triangolo), $S$. bifidus sp. n. (quadrato), S. kraussei sp. n. (triangolo vuoto), S. barbaricinus sp. n. (stella).

la precedente, stretta e ad apice acuto; lamella sinistra sovrapposta alla lamella mediana, ben più corta, larga alla base e gradatamente ristretta fino all'apice acuto; lamella posteriore larga alla base, arcuata all'apice (Fig. 28)

S. magrinii Bordoni, 2006

-. Lamella destra caratteristica per la forma stretta e lungamente arcuata (Fig. 29-30)

S. dorgaliensis sp. $\mathrm{n}$.

-. Edeago molto piccolo (lungo 0,75 mm), con lamelle più o meno della stessa forma e lunghezza, a parte quella destra che è molto più corta delle

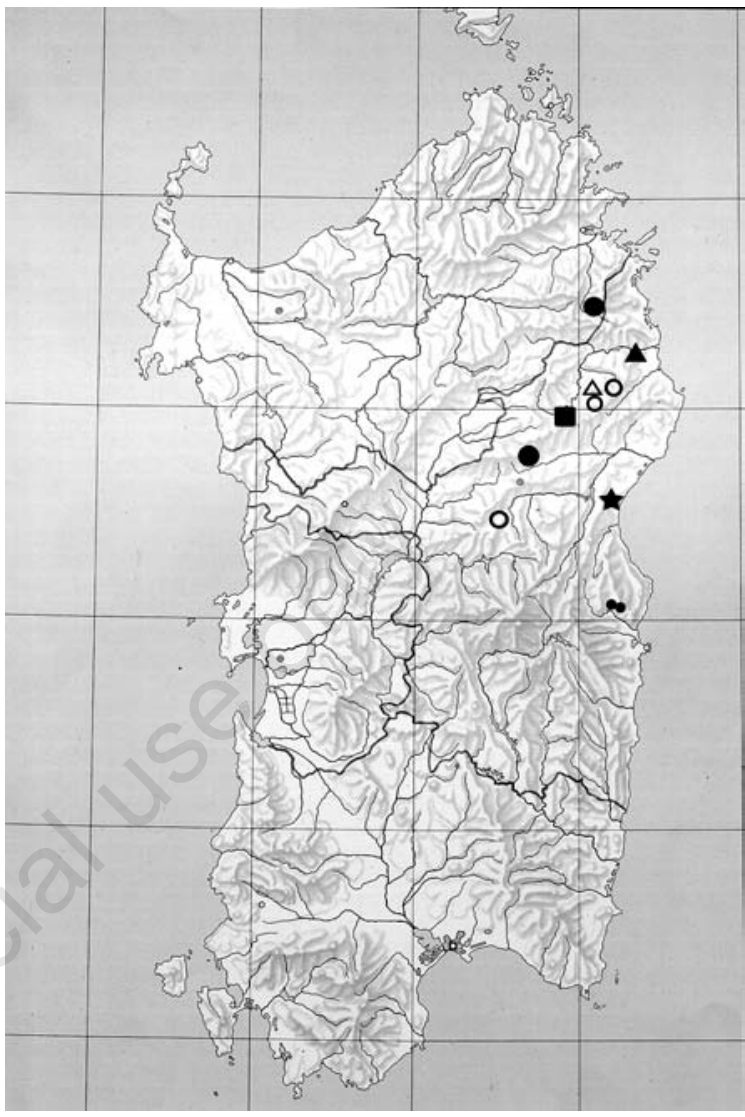

Fig. 44. Distribuzione degli Scotonomus del gruppo simpatricus: S. simpatricus Binaghi (stella), S. mirificus sp. n. (triangolo), S. baroniae sp. n. (triangolo vuoto), S. caneparii sp. n. (tondo grande), S. lulensis sp. n. (quadrato), S. nuragicus Bordoni (tondo piccolo), S. doderoi Binaghi (cerchio vuoto).

altre (Fig. 31)

S. saulcyi Coiffait, 1970

-. Lamella mediana robusta, più lunga delle altre, ad apice uncinato (Fig. 33); lamella posteriore a gomito e con apice fortemente uncinato (Fig. 34)

S. raymondi Fauvel, 1873

-. Lamelle lunghe e strette, più o meno della stessa lunghezza, a parte quella sinistra più corta e un poco sinuosa (Fig. 35); lamella posteriore fortemente uncinata (Fig. 36)

S. confusus sp. n.

-. Lamella mediana molto larga, a parte la porzione apicale vistosamente arcuata (Fig. 37); lamella si- 


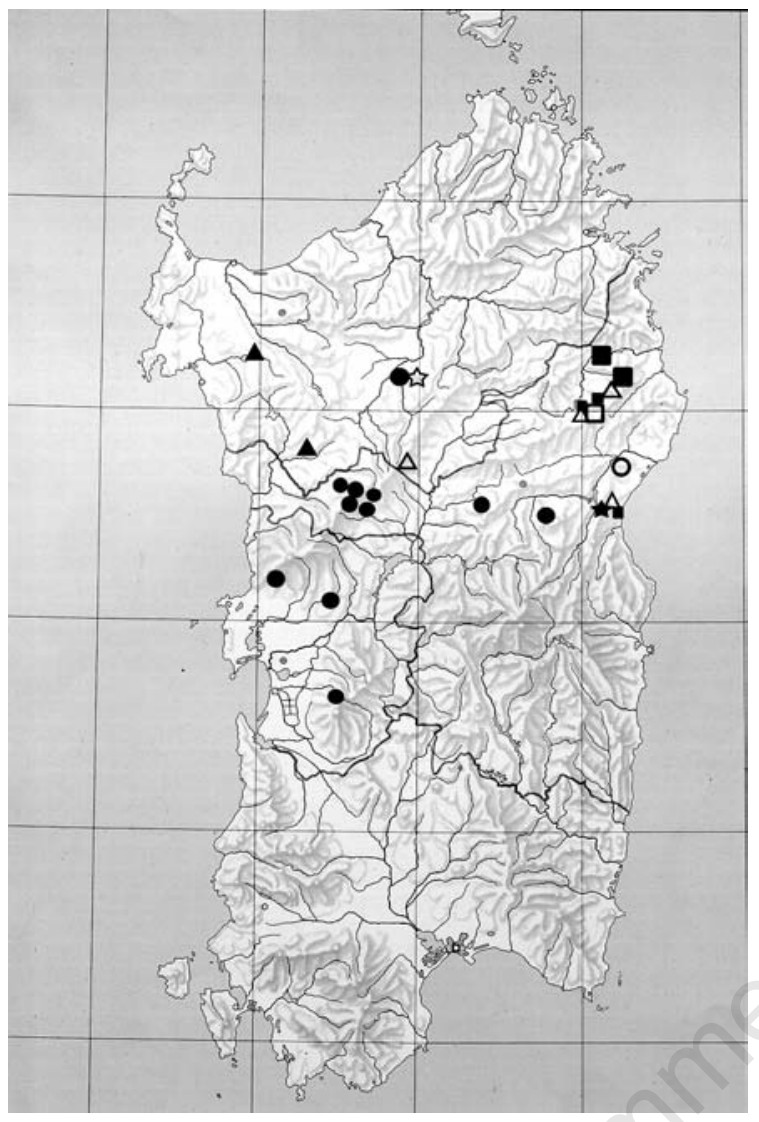

Fig. 45. Distribuzione degli Scotonomus del gruppo raymondi: S. consortii Bordoni \& Magrini (cerchio vuoto), $S$. magrinii Bordoni (quadrato), S. dorgaliensis sp. n. (stella piena), S. saulcyi Coiffait (triangolo pieno), S. raymondi Fauvel (stella vuota), S. confusus sp. n. (quadrato piccolo), S. montalbicus sp. n. (quadrato vuoto), S. binaghii Coiffait (tondo), S. fauveli sp. n. (triangolo vuoto).

nistra sovrapposta alla precedente, molto robusta; lamella posteriore un poco a gomito e ad apice vistosamente uncinato (Fig. 38)

\section{S. montalbicus sp. $\mathrm{n}$.}

-. Lamella mediana stretta, con porzione distale vistosamente a gomito (Fig. 39); lamella posteriore molto larga alla base, un poco arcuata all'apice che è subacuto (Fig. 40).

S. binaghii Coiffait, 1982

-. Lamella mediana larga, a parte la porzione distale, non a gomito, (Fig. 41); lamella posteriore

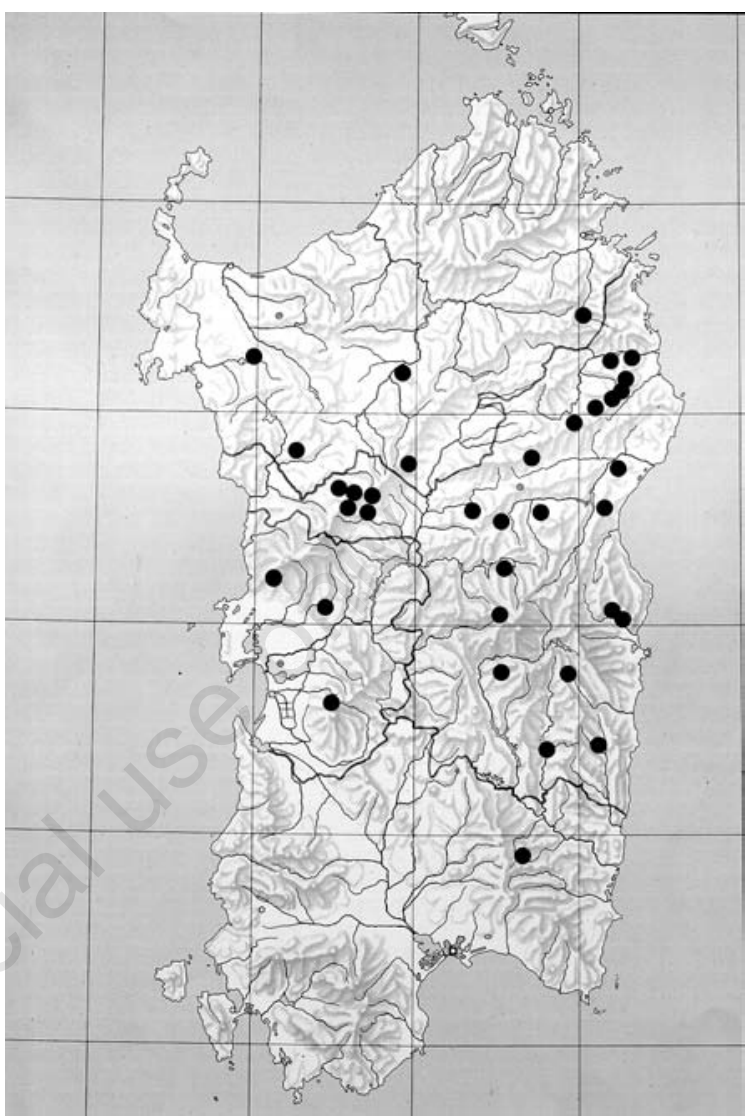

Fig. 46. Distribuzione generale degli Scotonomus Fauvel in Sardegna.

più stretta alla base che in binaghii e con apice spesso e fortemente uncinato (Fig. 42)

S. fauveli sp. n.

\section{RINGRAZIAMENTI}

Si ringraziano per l'invio di tipi, informazioni e per le ricerche effettuate i colleghi Luca Bartolozzi (Museo Zoologico "La Specola", Firenze), Giulio Cuccodoro (Muséum d'Histoire naturelle, Genève), Thierry Deuve e Azadeh Taghavian (Muséum national 
d'Histoire naturelles, Paris), Yvonnick Gérard (Institut royal Histoire naturelle, Bruxelles), Paolo Magrini (Firenze), Roberto Poggi (Museo civico Storia naturale "G. Doria", Genova), Fabrizio Rigato (Museo civico di Storia naturale, Milano), Harald Schillhammer (Naturhistorisches Museum, Vienna), Marc Tronquet (Molitg-les-Bains), Manfred Uhlig e Johannes Frisch
(Museum für Naturkunde der Humboldt-Universität, Berlin), Augusto Vigna Taglianti ed Emanuele Piattella (Museo di Zoologia dell'Università degli Studi "La Sapienza", Roma), Lothar Zerche (Deutsches Entomologisches Institut, Müncheberg), Alberto Zilli (Museo civico di Zoologia, Roma), Paolo Magrini ha cortesemente fornito la base cartografica.

\section{BIBLIOGRAFIA}

BinaGHi G., 1970 - Revisione degli Scotonomus. Analisi di un genere paleotirrenico (Coleoptera Staphylinidae). Bollettino della Società entomologica italiana, 102, 7-8: 118-136.

Bordoni A., 1975 - Scotonomus arganoi n. sp. del Lazio (Coleoptera, Staphylinidae). Fragmenta entomologica, 11: 103105.

Bordoni A., 2006 - Una nuova specie di Scotonomus del Monte Albo in Sardegna (Insecta, Coleoptera: Staphylinidae). Aldrovandia, 2: 7-10.

Bordoni A., 2010. Scotonomus nuragicus n. sp. from Sardinia (Coleoptera, Staphylinidae). Redia, 93: 109-110.

Bordoni A. \& P. Magrini, 2003 - Una nuova specie di Scotonomus della Sardegna (Coleoptera Staphylinidae). Bollettino della Società entomologica italiana, 135, 3: 167-171.

CASsola F., 1983 - L'esplorazione naturalistica della Sardegna. Lavori della Società italiana di Biogeografia, (n. s.) 8 (1980): 5-34.

CoIffait H., 1971 - Les Scotonomus, lignée endogée relique (Col. Staphylinidae). Description de quattre espèces nouvelles. Annales de Spéléologie, 25, 3: 725-733.

CoIffait H., 1982 - Coléoptères Staphylinidae de la Région paléartique occidentale. IV. Sous famille Paederinae. Tribu Paederini 1 (Paederi, Lathrobii). Nouvelle Revue d'Entomologie, suppl., 13, 4, 440 pp.

FAgel G., 1969 - Contribution à la connaissance des Staphylinidae. CVIII. Sur quelques espèces inédites provenant du bassin méditerranéen. Bulletin et Annales de la Societé Royale d'Entomologie de Belgique, 105: 103-125.

FAuvel A., 1873 - Faune Gallo-Rhénane ou species des insects qui habitent la France, la Belgique, la Hollande, le Luxenbourg, la Prusse rhénane, le Nassau e le Vallais avec tableau synoptiques et plaches gravées. Tome 3. Livraison 4. Caen: 215-390.

JEAnNel R., 1937 - Les Bembidiides endogés (Col. Carabidae). Monographie d'une lignée gondwanienne. Revue Française d'Entomologie, 3, 4: 241-399.

PACE R., 1977 - Quindici nuove specie di Stafilinidi ipogei dell'Italia centro-meridionale (Coleoptera). Redia, 60: $125-177$.

Zanetti A., 1977. Due nuove specie di Stafilinidi (Coleoptera) dell'Appennino. Bollettino del Museo Civico di Storia Naturale, Verona, 4: 307-315.

Indirizzo degli autori:

Arnaldo Bordoni, Museo di Storia naturale dell’Università, sezione di Zoologia “La Specola", via Romana 17, 50125

Firenze. E-mail: mailto:arnaldo.bordoni@libero.it

Luca Fancello, via Bainsizza 12,09123 Cagliari. E-mail: 1.fancello@hotmail.it

Piero Leo, via Tola 21, 09128 Cagliari. E-mail: piero.leo@tiscalinet.it 